\title{
Analyse par éléments-finis tridimensionnelle du comportement élastoplastique avec contact frottant
}

\author{
El Hassan Boudaia ${ }^{1,2, a}$, Lahbib Bousshine ${ }^{2}$, Ali ChaAba ${ }^{3}$, Hicham Fihri Fassi $^{4}$ \\ ET Gery De SAXCÉ ${ }^{5}$ \\ 1 Département génie mécanique, Faculté des Sciences et Techniques, Mahrila, BP 523, Beni Mellal 23000, Maroc \\ 2 Laboratoire des Technologies de Constructions et des Systèmes Industriels, ENSEM, BP 8118, Oasis, Casablanca, Maroc \\ 3 Laboratoire de Mécanique, École Nationale Supérieure des Arts et Métiers, BP 4024, Marjane II, Meknès, Maroc \\ 4 Département génie mécanique, Faculté des Sciences et Techniques, Settat, Maroc \\ ${ }^{5}$ Laboratoire de Mécanique, Université des Sciences et Technologies de Lille 1, 59655 Villeneuve d'Ascq Cedex, France
}

Reçu le 19 novembre 2008, accepté le 5 octobre 2009

Résumé - Dans cet article, nous présentons une analyse théorique et numérique des problèmes élastoplastiques incrémentaux basés sur la méthode des éléments-finis (MEF) et l'analyse numérique. L'étude est faite sur un matériau élastoplastique parfait obéissant au critère de von Mises. Le contact avec frottement sec de Coulomb à l'interface outil-pièce est pris en compte et formulé par la méthode du matériau standard implicite (MSI), proposée par De Saxcé et al., ce qui conduit à un seul principe variationnel de minimum en déplacement pour lequel le contact et le frottement sont couplés dans la fonction bipotentiel non différentiable en certains points. En vue de contourner cette difficulté nous proposons d'utiliser soit la méthode de régularisation soit le lagrangien augmenté avec l'algorithme de projection d'Uzawa. Par contre le problème de couplage est résolu par l'utilisation d'une procédure itérative basée sur la méthode du point fixe.

Mots clés : Élastoplasticité / méthode des éléments-finis / frottement / bipotentiel / méthode de régularisation / lagrangien augmenté / méthode du point fixe

Abstract - Three-dimensional finite-element analysis of elastoplastic behavior with frictional contact. In this paper, we present a theoretical and numerical analysis of the incremental elastoplastic problems based on the finite-element method (FEM) and the numerical analysis. The survey is made on a perfect elastoplastic material obeying the criteria of von Mises. The contact with Coulomb's dry friction to the tool-chip interface is taken into account and is formulated by the method of the implicit standard material (ISM), proposed by De Saxcé and al., that led to only one variationnal principle of minimum in displacement for which contact and friction are coupled in the bipotential function non differentiable in certain points. In order to overcome this difficulty we suggest the use whether out of the regularization method or the augmented Lagrangian with Uzawa's algorithm. On the other hand the problem of coupling is solved by the use of an iteration procedure based on the fixed point method.

Key words: Elastoplastic behavior / finite element method / friction / bipotential / regularization method / augmented lagrangian / fixed point method

\section{Introduction}

La modélisation des procédés de mise en forme par déformation plastique des pièces mécaniques en présence du contact avec frottement présente de nombreuses difficultés. Celles-ci proviennent des multiples non-linéarités mécaniques telles que : celles dues à la loi de comportement plastique du matériau constitutif de la pièce à

\footnotetext{
a Auteur pour correspondance :

Boudelhassan@yahoo.fr
}

déformer et celles introduites par la présence du contact avec frottement dans les interfaces entre la pièce à déformer et l'outil utilisé.

Notre attention est focalisée essentiellement sur la deuxième difficulté concernant les conditions aux limites de contact avec frottement. Par simplicité, nous adoptons la loi de contact unilatéral avec frottement sec de Coulomb, qui reste la plus utilisée dans ce type de problèmes.

Précisons que la loi de contact unilatéral avec frottement sec de Coulomb est une loi dissipative et irrégulière 


\section{Nomenclature}

\begin{tabular}{|c|c|}
\hline & \\
\hline$b_{\mathrm{c}}$ & Fonction bipotentiel \\
\hline$E$ & Module d'Young \\
\hline$e$ & Composantes du déviateur de déformations \\
\hline$e_{\mathrm{m}}$ & Trace du tenseur de déformations \\
\hline$f$ & Fonction de charge \\
\hline$G$ & Module de cisaillement de Coulomb \\
\hline$K_{\mathrm{c}}$ & Facteur de compressibilité \\
\hline$K_{\mu}$ & Cône de frottement de Coulomb \\
\hline$n$ & Vecteur unitaire normal \\
\hline$N(x)$ & Fonctions de formes \\
\hline$s$ & Composantes du déviateur de contraintes \\
\hline$s_{\mathrm{m}}$ & Trace du tenseur de contraintes \\
\hline$t$ & Action de contact \\
\hline$u$ & Déplacement \\
\hline$u^{\mathrm{f}}$ & Déplacement fictif \\
\hline$\dot{u}$ & Vecteur vitesse de déplacement \\
\hline$V(\varepsilon)$ & Surpotentiel de déformation et sa polaire $W(\sigma)$ \\
\hline \multicolumn{2}{|c|}{ Symboles grecs } \\
\hline$\varepsilon$ & Déformation \\
\hline$\varepsilon^{\mathrm{e}}$ & Déformation élastique \\
\hline$\varepsilon^{\mathrm{p}}$ & Déformation plastique \\
\hline$\dot{\varepsilon}$ & Champ de vitesses de déformations \\
\hline$\mu$ & Coefficient de frottement \\
\hline$\sigma$ & Champ de contrainte \\
\hline$\sigma_{Y}$ & Contrainte d'écoulement \\
\hline$\Omega$ & Structure (volume du corps) \\
\hline$\Delta b_{\mathrm{c}}$ & Incrément du bipotentiel de contact \\
\hline$\Delta F$ & Incréments de forces généralisées \\
\hline$\Delta \bar{f}$ & Force imposée par unité de volume \\
\hline$\Delta K_{\mu}$ & Incréments de traction admissibles \\
\hline$\Delta \bar{t}$ & Tractions imposées par unité de surface \\
\hline$\Delta t_{\mathrm{n}}$ & Incrément de contrainte normale \\
\hline$\Delta t_{\mathrm{t}}$ & Incrément de contrainte tangentielle \\
\hline$\Delta u$ & Incrément de déplacement \\
\hline$\Delta \bar{u}$ & Déplacements imposés \\
\hline$\Delta V$ & Surpotentiel de déformation incrémental \\
\hline$\Delta V_{\mathrm{e}}$ & Surpotentiel incrémental élastique \\
\hline$\Delta V_{\mathrm{p}}$ & Surpotentiel incrémental plastique \\
\hline$\Delta \beta$ & Incrément de la fonction bifonctionnelle \\
\hline$\Delta \varepsilon$ & Incrément de déformation \\
\hline$\Delta \sigma$ & Incrément de contrainte \\
\hline$\partial K_{\mu}$ & Surface de glissement \\
\hline$\otimes$ & Produit de convolution \\
\hline$\|\|$. & Norme euclidienne \\
\hline
\end{tabular}

faisant intervenir trois statuts tels que, non contact, contact avec adhérence et contact avec glissement. En outre, ce phénomène est similaire au comportement rigide parfaitement plastique dans le sens suivant : le critère de Coulomb est analogue à celui de plasticité et la loi de glissement à la règle d'écoulement. Sur la base de cette idée, la théorie des surpotentiels de dissipation, décrivant les comportements plastiques des matériaux [1,2], constitue un meilleur cadre de comparaison de ces lois qui apparaissent tellement différentes.

Sur la base de la théorie des matériaux standards implicites (MSI), introduite par de Saxcé et ses collaborateurs [3], la loi de contact unilatéral avec frottement sec de Coulomb est écrite sous forme d'une loi de normalité implicite. Dans le cadre théorique des MSI, la notion du bipotentiel [4] conduit à l'introduction de nouveaux principes variationnels basés sur une fonctionnelle dépendant des deux champs de déplacement et de contrainte, dite bifonctionnelle. Pour le contact unilatéral avec frottement, le modèle des MSI conduit à un seul principe variationnel de minimum en déplacement pour lequel le contact et le frottement sont couplés dans la fonction bipotentiel de contact. Cela justifie l'utilisation de l'approche des MSI dans ce présent travail.

Nous signalons que nous sommes dans le contexte d'un problème d'évolution élastoplastique que nous devons résoudre de façon incrémentale à cause de son caractère non-linéaire, en appliquant un schéma d'intégration 
implicite sur le temps aux lois de comportement temporelles pour obtenir d'une part la formulation incrémentale et d'autre part pour satisfaire la stabilité du critère de frottement à la fin du pas.

Pour la résolution numérique, la détermination de l'incrément de déplacement par minimisation de la fonctionnelle incrémentale exige l'utilisation d'un code de calcul de la programmation mathématique. Cependant, le code adopté (Minos [5]) demande la connaissance des gradients de la fonction objectif. Cette dernière contient un terme non différentiable représentant le bipotentiel de contact unilatéral avec frottement sec de Coulomb. Cela se manifeste aussi au cours du calcul de l'incrément de la réaction de contact par la minimisation du bipotentiel.

En vue de surmonter cette difficulté nous proposons d'utiliser deux méthodes de natures différentes : la méthode de régularisation par pénalisation et le lagrangien augmenté avec l'algorithme de projection d'Uzawa [6], parce que la méthode dite du lagrangien augmenté a été proposée en premier par Hestenss [7] et Powell [8], et utilisée par la suite dans plusieurs travaux [9] sur le contact. En particulier, Feng et al. [10] ont utilisé le lagrangien augmenté avec l'algorithme d'Uzawa pour résoudre le problème de contact avec frottement dans le cas d'un corps élastique linéaire. En plus, la considération du comportement de l'interface de contact comme MSI nous a permis de résoudre les équations de contact et de frottement par une seule étape prédiction/correction au lieu de deux étapes proposées par Jean et Touzot [11].

Une deuxième difficulté qui ne manque pas d'importance est la présence d'un terme de couplage dans la fonctionnelle incrémentale à minimiser par la considération du principe variationnel de déplacement. Ce terme de couplage se trouve dans le bipotentiel de contact et de frottement qui contient les forces de traction normales de contact. Nous précisons que ces dernières constituent des variables statiques dont les valeurs ne sont pas encore déterminées. Ce problème de couplage est résolu par l'utilisation d'une procédure itérative basée sur la méthode du point fixe.

Dans ce travail, la méthode des éléments-finis cinématiquement admissible est choisie pour discrétiser le champ de déplacement incrémental. Nous nous basons sur cette méthode numérique et la programmation mathématique en vue de traiter des problèmes de la mise en forme des métaux tel que le poinçonnement...

\section{Formulation locale des conditions de contact}

Les équations du contact local se décomposent en deux parties :

- des conditions sur l'aspect unilatéral du contact appelé conditions de Signorini traduisant l'impossibilité d'interpénétrabilité de corps déformables [12] ;

- des conditions sur la composante tangentielle des contraintes sur la surface de contact dont un exemple est la loi de frottement sec de Coulomb.
Considérons une structure $\Omega$ composée de deux solides disjoints $\Omega_{\mathrm{A}}$ et $\Omega_{\mathrm{B}}$ séparés par une interface $S_{\mathrm{c}}$ qui peut être une surface de contact au niveau du plan tangent commun $\pi\left(t_{t x}, t_{t y}\right)$, orthogonal au vecteur unitaire normal $n$. Les vecteurs vitesses relatives $\dot{u}$ et l'action de contact $t$ peuvent être décomposés de manière unique sous la forme suivante :

$$
\left\{\begin{array}{l}
\dot{u}=\dot{u}_{\mathrm{t}}+\dot{u}_{\mathrm{n}} n, \dot{u}_{\mathrm{t}} \in \pi \text { et } \dot{u}_{\mathrm{n}} \in \mathbb{R} \\
t=t_{\mathrm{t}}+t_{\mathrm{n}} n, t_{\mathrm{t}} \in \pi \text { et } t_{\mathrm{n}} \in \mathbb{R} \text { avec }\left\|t_{\mathrm{t}}\right\|=\sqrt{t_{t x}^{2}+t_{t y}^{2}}
\end{array}\right.
$$

En combinant la loi de contact unilatéral et de frottement sec de Coulomb, nous pouvons écrire que ( $\mu$ désigne le coefficient de frottement) :

$$
\left\{\begin{array}{l}
\text { si } t_{\mathrm{n}}=0 \text { alors }-\dot{u}_{\mathrm{n}} \leq 0 \text { relâchement } \\
\text { sinon si } t_{\mathrm{n}}>0 \text { et }\left\|t_{\mathrm{t}}\right\|<\mu t_{\mathrm{n}}, \\
\text { alors } \dot{u}_{\mathrm{n}}=0, \dot{u}_{\mathrm{t}}=0 \text { adhérence } \\
\text { sinon }\left(t_{\mathrm{n}}>0 \text { et }\left\|t_{\mathrm{t}}\right\|=\mu t_{\mathrm{n}}\right),\left\{\dot{u}_{\mathrm{n}}=0 \text { et } \exists \dot{\lambda} \geqslant 0\right. \\
\text { tel que } \left.-\dot{u}_{\mathrm{t}}=\dot{\lambda} \frac{t_{\mathrm{t}}}{\left\|t_{\mathrm{t}}\right\|}\right\} \text { glissement }
\end{array}\right.
$$

La loi inverse s'écrit sous la forme suivante :

$$
\left\{\begin{array}{l}
\text { si } \dot{u}_{\mathrm{n}}>0 \text { alors } t=0 \text { relâchement } \\
\text { sinon si } \dot{u}=0 \text { alors }\left\|t_{\mathrm{t}}\right\|<\mu t_{\mathrm{n}} \text { adhérence } \\
\text { sinon } \dot{u}_{\mathrm{t}} \neq 0, \dot{u}_{\mathrm{n}}=0 \text { et } t_{\mathrm{t}}=-\mu t_{\mathrm{n}} \frac{\dot{u}_{\mathrm{t}}}{\left\|\dot{u}_{\mathrm{t}}\right\|} \text { glissement }
\end{array}\right.
$$

Le cône de frottement de Coulomb est défini par l'ensemble :

$$
K_{\mu}=\left\{\left(t_{\mathrm{n}}, t_{\mathrm{t}}\right) \text { tel que }\left\|t_{\mathrm{t}}\right\|-\mu t_{\mathrm{n}} \leqslant 0\right\}
$$

Le bipotentiel de contact avec frottement est défini par [4] :

$$
b_{\mathrm{c}}(-\dot{u}, t)=\left\{\begin{array}{l}
\mu t_{\mathrm{n}}\left\|-\dot{u}_{\mathrm{t}}\right\| \text { si } t \in K_{\mu} \text { et } \dot{u}_{\mathrm{n}} \geq 0 \\
+\infty \text { sinon }
\end{array}\right.
$$

En résumé, la loi de contact complète et sa loi inverse peuvent s'écrire respectivement sous formes de lois de sous normalité implicite [10] :

$$
-\dot{u} \in \partial_{\mathrm{t}} b_{\mathrm{c}}(-\dot{u}, t) \text { et } t \in \partial_{-\dot{u}} b_{\mathrm{c}}(-\dot{u}, t)
$$

De ce qui précède, nous pouvons conclure que le concept $\mathrm{du}$ bipotentiel permet d'écrire de façon claire et sans découplage entre les paramètres de contact et de frottement.

\section{Problème d'évolution élastoplastique}

Le comportement élastoplastique des métaux est supposé obéir au modèle de Saint Venant qui est basé sur l'hypothèse de décomposition des déformations $\varepsilon$ en deux parties :

$$
\varepsilon=\varepsilon^{\mathrm{e}}+\varepsilon^{\mathrm{p}}
$$




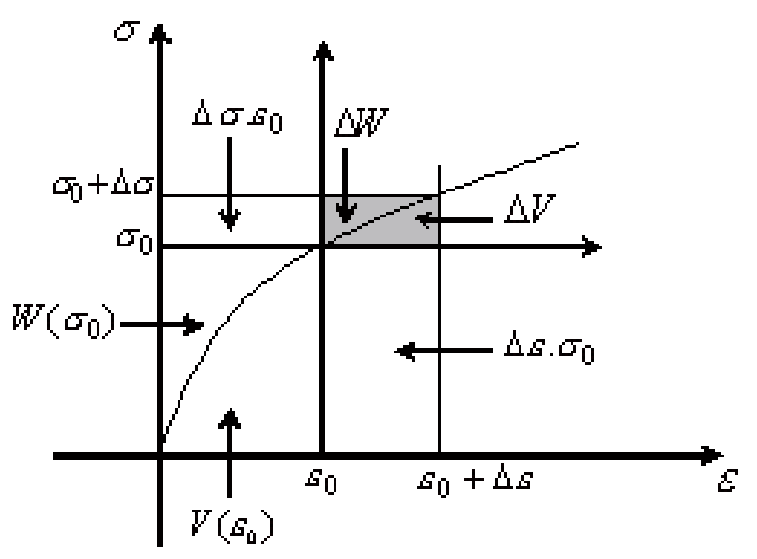

Fig. 1. Courbe contrainte-déformation.

où $\varepsilon^{\mathrm{e}}$ est la déformation élastique (réversible) obéissant à la loi de Hooke décrite par la relation : $\varepsilon^{\mathrm{e}}=S \sigma(S$ désigne la matrice des souplesses élastiques) et $\varepsilon^{\mathrm{p}}$ est la déformation plastique (irréversible) donnée par :

$$
\dot{\varepsilon}^{\mathrm{p}}=\dot{\lambda} \frac{\partial f}{\partial \sigma}
$$

où $\lambda$ est un coefficient de proportionnalité strictement positif et $f$ la fonction de charge.

\subsection{Schéma d'intégration des lois constitutives}

La loi incrémentale permet de transformer un problème non linéaire par un autre linéaire par morceaux. Soit un matériau standard défini par son surpotentiel $V(\varepsilon)$ et sa polaire $W(\sigma)$, (voir Fig. 1) :

Considérons les notations suivantes :

$$
\Delta \sigma=\sigma_{1}-\sigma_{0} ; \Delta \varepsilon=\varepsilon_{1}-\varepsilon_{0} ; \Delta \varepsilon^{\mathrm{e}}=\varepsilon_{1}^{\mathrm{e}}-\varepsilon_{0}^{\mathrm{e}} ; \Delta \varepsilon^{\mathrm{p}}=\varepsilon_{1}^{\mathrm{p}}-\varepsilon_{0}^{\mathrm{p}}
$$

où l'index 0 (respectivement 1) est relatif au début (resp. à la fin) du pas.

Nous pouvons alors définir le surpotentiel de déformation incrémental par :

$$
\Delta V(\Delta \varepsilon)=V\left(\varepsilon_{0}+\Delta \varepsilon\right)-V\left(\varepsilon_{0}\right)-\Delta \varepsilon \sigma_{0}
$$

et nous pouvons montrer facilement que sa fonction polaire appelée surpotentiel complémentaire incrémental, est égale à :

$$
\Delta W(\Delta \sigma)=W\left(\sigma_{0}+\Delta \sigma\right)-W\left(\sigma_{0}\right)-\Delta \sigma \varepsilon_{0}
$$

\subsection{Surpotentiel élastoplastique incrémental}

Dans ce paragraphe, nous allons étendre le concept de l'inf-convolution [13] au calcul du surpotentiel incrémental. Soient $V_{1}\left(x_{1}\right)$ et $V_{2}\left(x_{2}\right)$ deux surpotentiels, nous introduisons une nouvelle fonction notée par :

$$
V=V_{1} \otimes_{x} V_{2}
$$

appelée inf-convolution de $V_{1}$ et $V_{2}$ par rapport à la variable $x$ et définie par :

$$
V(x)=\operatorname{Inf}_{x^{\prime}}\left(V_{1}\left(x-x^{\prime}\right)+V_{2}\left(x^{\prime}\right)\right)
$$

Théorème : L'inf-convolution de deux surpotentiels est un surpotentiel.

Preuve : Soit $W(y)$ la fonction polaire de $V(x)$ au sens de Fenchel. Nous pouvons vérifier facilement la condition de convexité d'où :

$$
\left\{\begin{array}{l}
\forall x_{1}, y, V_{1}\left(x_{1}\right)+W_{1}(y) \geq x_{1} y \\
\forall x_{2}, y, V_{2}\left(x_{2}\right)+W_{2}(y) \geq x_{2} y
\end{array}\right.
$$

En additionnant les deux inéquations ci-dessus, membre à membre, nous obtenons :

$$
\forall x, x^{\prime}, y, \operatorname{Inf}\left(V_{1}\left(x-x^{\prime}\right)+V_{2}\left(x^{\prime}\right)\right)+W(y) \geq x y
$$

Ainsi, il est prouvé que l'inf-convolution est un surpotentiel.

De ce qui précède, nous pouvons introduire le surpotentiel élastoplastique incrémental :

$$
\begin{aligned}
& \Delta V(\Delta \varepsilon)=\left(\Delta V_{\mathrm{e}} \otimes \Delta V_{\mathrm{p}}\right)(\Delta \varepsilon) \\
& \quad=\operatorname{Inf}_{\Delta \varepsilon^{\mathrm{p}} \text { admissible }}\left(\Delta V_{\mathrm{e}}\left(\Delta \varepsilon-\Delta \varepsilon^{\mathrm{p}}\right)+\Delta V_{\mathrm{p}}\left(\Delta \varepsilon^{\mathrm{p}}\right)\right)
\end{aligned}
$$

et sa fonction polaire :

$$
\Delta W=\Delta W_{\mathrm{e}}+\Delta W_{\mathrm{p}}
$$

où $\Delta V_{\mathrm{e}}$ et $\Delta W_{\mathrm{e}}$ représentent les surpotentiels incrémentaux élastiques et $\Delta V_{\mathrm{p}}$ et $\Delta W_{\mathrm{p}}$ désignent les surpotentiels incrémentaux plastiques.

\subsection{Schéma d'intégration implicite appliqué à l'élastoplasticité}

\subsection{1 Élastoplasticité en chargement uniaxial}

La loi élastoplastique parfaite suppose que le surpotentiel incrémental est composé de deux surpotentiels, l'un élastique $\Delta V_{\mathrm{e}}$ et l'autre plastique $\Delta V_{\mathrm{p}}$, le surpotentiel incrémental élastique $\Delta V_{\mathrm{e}}$ étant défini comme l'énergie de déformation élémentaire élastique donnée par :

$$
\Delta V_{\mathrm{e}}\left(\Delta \varepsilon^{\mathrm{e}}\right)=\frac{1}{2} E\left(\Delta \varepsilon^{\mathrm{e}}\right)^{2}=\frac{1}{2} E\left(\Delta \varepsilon-\Delta \varepsilon^{\mathrm{p}}\right)^{2}
$$

Le surpotentiel incrémental de dissipation plastique $\Delta V_{\mathrm{p}}$ est donné par :

$$
\Delta V_{\mathrm{p}}\left(\Delta \varepsilon^{\mathrm{p}}\right)=\sigma_{Y}\left|\Delta \varepsilon^{\mathrm{p}}\right|-\sigma_{0} \Delta \varepsilon^{\mathrm{p}}
$$

où $\sigma_{Y}$ est la contrainte d'écoulement et $E$ le module de Young.

Le surpotentiel élastoplastique incrémental (16) devient :

$$
\Delta V(\Delta \varepsilon)=\operatorname{Inf}_{\Delta \varepsilon^{\mathrm{p}}}\left(\frac{1}{2} E\left(\Delta \varepsilon-\Delta \varepsilon^{\mathrm{p}}\right)^{2}+\sigma_{Y}\left|\Delta \varepsilon^{\mathrm{p}}\right|-\sigma_{0} \Delta \varepsilon^{\mathrm{p}}\right)
$$


Remarquons que la fonction à minimiser dans (20) n'est pas différentiable au point $\Delta \varepsilon^{\mathrm{p}}=0$. Dans ce cas, nous avons $\Delta \sigma=E \Delta \varepsilon^{\mathrm{e}}$.

La non différentiabilité du surpotentiel $\Delta V_{\mathrm{p}}$ n'a plus d'effet sur le calcul du surpotentiel $\Delta V$ grâce à la présence du surpotentiel différentiable $\Delta V_{\mathrm{e}}$. Il est à rappeler que nous avons utilisé la propriété de régularisation du concept de l'inf-convolution [13]. Le surpotentiel plastique est régularisé par le surpotentiel élastique. Dans les autres cas, la condition de stationnarité donne :

$$
E\left(\Delta \varepsilon^{\mathrm{p}}-\Delta \varepsilon\right)+\operatorname{sign}\left(\Delta \varepsilon^{\mathrm{p}}\right) \sigma_{Y}-\sigma_{0}=0
$$

Introduisons la prédiction élastique $\sigma_{1}^{*}=\sigma_{0}+E \Delta \varepsilon$. L'équation (21) devient :

$$
\operatorname{sign}\left(\Delta \varepsilon^{\mathrm{p}}\right)\left(E\left|\Delta \varepsilon^{\mathrm{p}}\right|+\sigma_{Y}\right)=\sigma_{1}^{*}
$$

La résolution de l'équation non-linéaire (18) se fait alors en deux étapes :

- le terme entre parenthèses $\left(E\left|\Delta \varepsilon^{\mathrm{p}}\right|+\sigma_{Y}\right)$ est positif d'où : $\operatorname{sign}\left(\Delta \varepsilon^{\mathrm{p}}\right)=\operatorname{sign}\left(\sigma_{1}^{*}\right)$;

- l'équation (22) prend la forme suivante : $E\left|\Delta \varepsilon^{\mathrm{p}}\right|+$ $\sigma_{Y}=\left|\sigma_{1}^{*}\right|$ qui donne immédiatement la valeur de l'incrément plastique :

$$
\left|\Delta \varepsilon^{\mathrm{p}}\right|=\frac{1}{E}\left(\left|\sigma_{1}^{*}\right|-\sigma_{Y}\right)
$$

Cette égalité a un sens sauf si la prédiction élastique n'est pas plastiquement admissible c'est-à-dire que $\left|\sigma_{1}^{*}\right|>\sigma_{Y}$.

Dans le cas échéant, l'équation n'a pas de solution et le chargement est purement élastique. Si l'écoulement plastique se produit, la solution finale est obtenue en combinant signe et valeur absolue :

$$
\begin{aligned}
\Delta \varepsilon^{\mathrm{p}} & =\operatorname{sign}\left(\Delta \varepsilon^{\mathrm{p}}\right)\left|\Delta \varepsilon^{\mathrm{p}}\right|=\operatorname{sign}\left(\sigma_{1}^{*}\right)\left(\left|\sigma_{1}^{*}\right|-\sigma_{Y}\right) / E \\
& =\frac{1}{E}\left(\sigma_{1}^{*}-\operatorname{sign}\left(\sigma_{1}^{*}\right) \sigma_{Y}\right)
\end{aligned}
$$

La valeur de l'incrément de la contrainte est obtenue par :

$$
\Delta \sigma=E\left(\Delta \varepsilon-\Delta \varepsilon^{\mathrm{p}}\right)=\operatorname{sign}\left(\sigma_{1}^{*}\right) \sigma_{Y}-\sigma_{0}
$$

En résumé, nous obtenons le schéma prédiction/correction suivant :

Prédiction :

$$
\text { calculer : } \sigma_{1}^{*}=\sigma_{0}+E \Delta \varepsilon
$$

\section{Correction :}

si $\left|\sigma_{1}^{*}\right| \leqslant \sigma_{Y}$ alors $\Delta \varepsilon^{\mathrm{p}}=0$ et $\Delta \sigma=E \Delta \varepsilon^{\mathrm{e}}$ : charge ou décharge élastique

sinon $\Delta \varepsilon^{\mathrm{p}}=\frac{1}{E}\left(\sigma_{1}^{*}-\operatorname{sign}\left(\sigma_{1}^{*}\right) \sigma_{Y}\right):$ chargement élastoplastique

$$
\Delta \sigma=\operatorname{sign}\left(\sigma_{1}^{*}\right) \sigma_{Y}-\sigma_{0}
$$

De ce qui précède, nous obtenons l'expression finale du surpotentiel élastoplastique incrémental différentiable partout :

$$
\Delta V(\Delta \varepsilon)=\frac{E}{2}\left((\Delta \varepsilon)^{2}-\frac{1}{E^{2}}\left(\left|E \Delta \varepsilon+\sigma_{0}\right|-\sigma_{0}\right)^{2}\right)
$$

L'incrément de la contrainte est obtenu à partir de la dérivée explicite de $\Delta V(\Delta \varepsilon)$ :

$$
\begin{aligned}
\Delta \sigma & =\frac{\partial \Delta V(\Delta \varepsilon)}{\partial \Delta \varepsilon} \\
& =E \Delta \varepsilon-\left(\sigma_{1}^{*}-\operatorname{sign}\left(\sigma_{1}^{*}\right) \sigma_{Y}\right) \Delta\left(\left|\sigma_{1}^{*}\right|-\sigma_{Y}\right)
\end{aligned}
$$

En particulier, si $\left|\sigma_{1}^{*}\right|<\sigma_{Y}$ nous avons $\Delta \sigma=E \Delta \varepsilon^{\mathrm{e}}$, sinon $\Delta \sigma=\operatorname{sign}\left(\sigma_{1}^{*}\right) \sigma_{Y}-\sigma_{0}$.

\subsection{2 Élastoplasticité en chargement multiaxial}

Tenant compte de la décomposition des tenseurs de déformations et de contraintes en parties déviatorique et sphérique et de la déformation plastique incompressible pour les métaux, nous adoptons les variables suivantes :

$$
\varepsilon \equiv\left(e_{\mathrm{m}}, e\right) ; \varepsilon^{\mathrm{p}} \equiv\left(e_{\mathrm{m}}^{\mathrm{p}}, e^{\mathrm{p}}\right) \equiv\left(0, e^{\mathrm{e}}\right) \text { et } \sigma \equiv\left(s_{\mathrm{m}}, s\right)
$$

où $\left(e_{\mathrm{m}}, s_{\mathrm{m}}\right)$ et $(e, s)$ sont respectivement les traces des tenseurs et les composantes des déviateurs de déformations et de contraintes.

Le surpotentiel incrémental élastique $\Delta V_{\mathrm{e}}$ est défini par :

$$
\begin{aligned}
\Delta V_{\mathrm{e}} & =\frac{1}{2} \Delta \varepsilon^{\mathrm{e}} \Delta \sigma=\frac{1}{2} \Delta \varepsilon^{\mathrm{e}} S^{-1} \Delta \varepsilon^{\mathrm{e}} \\
& =\frac{K_{\mathrm{c}}}{2}\left(\Delta e_{\mathrm{m}}^{\mathrm{e}}\right)^{2}+G\left\|\Delta e^{\mathrm{e}}\right\|^{2}
\end{aligned}
$$

où $K_{\mathrm{c}}$ et $G$ sont respectivement le facteur de compressibilité et le module de cisaillement de Coulomb, leurs expressions sont données en fonction du coefficient de Poisson $\nu$ et module de Young $E$ :

$$
K_{\mathrm{c}}=\frac{E}{3(1-2 \nu)} \text { et } G=\frac{E}{2(1+\nu)}
$$

Nous rappelons que le surpotentiel plastique du critère de von Mises est calculé par l'expression suivante :

$$
\begin{aligned}
& V_{\mathrm{p}}\left(\dot{e}^{\mathrm{p}}\right)=\sqrt{\frac{2}{3}} \sigma_{Y}\left\|\dot{e}^{\mathrm{p}}\right\|=\sigma_{Y} \dot{p}\left(\dot{e}^{\mathrm{p}}\right) \\
& \dot{p}\left(\dot{e}^{\mathrm{p}}\right)=\sqrt{\frac{2}{3} \dot{e}^{\mathrm{p}}: \dot{e}^{\mathrm{p}}}
\end{aligned}
$$

Donc, le surpotentiel incrémental plastique $\Delta V_{\mathrm{p}}$ sera déterminé par la dissipation plastique élémentaire comme suit :

$$
\Delta V_{\mathrm{p}}\left(\Delta \varepsilon^{\mathrm{p}}\right)=\sigma_{Y} p\left(\Delta e^{\mathrm{p}}\right)-s_{0} \Delta e^{\mathrm{p}}
$$

Si nous supposons que $\Delta e^{\mathrm{p}} \neq 0$, la condition de stationnarité s'écrit :

$$
2 G\left(\Delta e^{\mathrm{p}}-\Delta e\right)+\frac{2 \Delta e^{\mathrm{p}}}{3 p\left(\Delta e^{\mathrm{p}}\right)} \sigma_{Y}-s_{0}=0
$$

Introduisons la prédiction élastique :

$$
s_{1}^{*}=s_{0}+2 G \Delta e=\frac{2 \Delta e^{\mathrm{p}}}{3 p\left(\Delta e^{\mathrm{p}}\right)}\left(3 G p\left(\Delta e^{\mathrm{p}}\right)+\sigma_{Y}\right)
$$


La résolution de cette équation non-linéaire se fait alors en deux étapes :

- le terme entre parenthèses $\left(3 G p\left(\Delta e^{\mathrm{p}}\right)+\sigma_{Y}\right)$ est positif, d'où nous déduisons la direction de l'incrément de déformation plastique, donné par celui de la prédiction élastique :

$$
\frac{2}{3} \frac{\Delta e^{\mathrm{p}}}{p\left(\Delta e^{\mathrm{p}}\right)}=\frac{s_{1}^{*}}{\sigma_{\mathrm{eq}}\left(s_{1}^{*}\right)}
$$

- l'équation (34) prend la forme suivante :

$$
3 G p\left(\Delta e^{\mathrm{p}}\right)+\sigma_{Y}=\sigma_{\mathrm{eq}}\left(s_{1}^{*}\right)
$$

qui donne immédiatement la valeur de l'intensité de l'incrément :

$$
p\left(\Delta e^{\mathrm{p}}\right)=\frac{1}{3 G}\left(\sigma_{\mathrm{eq}}\left(s_{1}^{*}\right)-\sigma_{Y}\right)
$$

Cette égalité a un sens sauf si la prédiction élastique n'est pas plastiquement admissible c'est-à-dire que $\sigma_{\text {eq }}\left(s_{1}^{*}\right)>$ $\sigma_{Y}$.

Dans le cas échéant, l'équation n'a pas de solution et le chargement est purement élastique. Si l'écoulement plastique se produit, la solution finale est obtenue en combinant la direction définie par (35) et l'intensité fournie $\operatorname{par}(37)$ :

$$
\Delta e^{\mathrm{p}}=p\left(\Delta e^{\mathrm{p}}\right) \frac{\Delta e^{\mathrm{p}}}{p\left(\Delta e^{\mathrm{p}}\right)}=\frac{1}{2 G}\left(s_{1}^{*}-\sigma_{Y} \frac{s_{1}^{*}}{\sigma_{\mathrm{eq}}\left(s_{1}^{*}\right)}\right)
$$

La valeur de l'incrément de la contrainte est obtenue par :

$$
\Delta s=2 G\left(\Delta e-\Delta e^{\mathrm{p}}\right)=\sigma_{Y} \frac{s_{1}^{*}}{\sigma_{\mathrm{eq}}\left(s_{1}^{*}\right)}-s_{0}
$$

En résumé, nous obtenons le schéma prédiction/correction suivant :

\section{Prédiction :}

calculer $\Delta s_{\mathrm{m}}=K_{\mathrm{c}} \Delta e_{\mathrm{m}}$ et $s_{1}^{*}=s_{0}+2 G \Delta e$

Correction :

si $\sigma_{\text {eq }}\left(s_{1}^{*}\right) \leq \sigma_{Y}$ alors $\Delta e^{\mathrm{p}}=0$ et $\Delta s=2 G \Delta e$ : charge ou décharge élastique

sinon $\Delta e^{\mathrm{p}}=\frac{1}{2 G}\left(s_{1}^{*}-\sigma_{Y} \frac{s_{1}^{*}}{\sigma_{\text {eq }}\left(s_{1}^{*}\right)}\right):$ chargement élastoplastique

$$
\Delta s=\sigma_{Y} \frac{s_{1}^{*}}{\sigma_{\mathrm{eq}}\left(s_{1}^{*}\right)}-s_{0}
$$

De ce qui précède, nous obtenons l'expression finale du surpotentiel élastoplastique incrémental en terme de déformations, pour un matériau obéissant au critère de von Mises :

$$
\Delta V(\Delta \varepsilon)=\frac{1}{2} K_{\mathrm{c}}\left(\Delta e_{\mathrm{m}}\right)^{2}+G\left(\|\Delta e\|^{2}-\left\|\Delta e^{\mathrm{p}}\right\|^{2}\right)
$$

$$
\text { avec }\left\|\Delta e^{\mathrm{p}}\right\|=\|\Delta e\|-\frac{\sigma_{Y}}{G \sqrt{6}} \text { si }\|\Delta e\| \geq \frac{\sigma_{Y}}{G \sqrt{6}} \text {. }
$$

Enfin, les lois constitutives du comportement plastique du matériau s'écrivent :

$$
\Delta \varepsilon=\frac{\partial \Delta W(\Delta \sigma)}{\partial \Delta \sigma} \text { et } \Delta \sigma=\frac{\partial \Delta V(\Delta \varepsilon)}{\partial \Delta \varepsilon}
$$

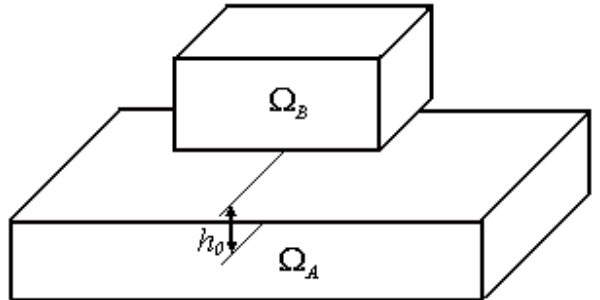

Fig. 2. Structure tridimensionnelle en contact.

\section{Schéma implicite appliqué au problème de contact frottement sec de Coulomb}

Considérons le contact entre deux solides $\Omega_{\mathrm{A}}$ et $\Omega_{\mathrm{B}}$ où l'un peut être une fondation rigide. Nous supposons que le contact peut se produire entre deux points $x_{\mathrm{A}}$ et $x_{\mathrm{B}}$ des deux solides espacés initialement de $h_{0}$ comme l'indique la figure 2 .

La loi de contact unilatéral avec frottement sec de Coulomb est similaire à la loi rigide parfaitement plastique. Pour les mêmes raisons citées à la section 3.4, le schéma d'intégration implicite, développé par Feng et De Saxcé [14], est utilisé.

Introduisons les notations suivantes :

$$
\Delta u=u_{1}-u_{0} ; \Delta t=t_{1}-t_{0}
$$

où les indices 0 et 1 désignent respectivement le début et la fin du pas.

Le schéma d'intégration implicite conduit à :

$$
\Delta u=u_{1}-u_{0}=\Delta \tau \dot{u}_{1}
$$

où $\dot{u}_{1}$ est la vitesse de glissement et $\Delta \tau$ l'incrément du temps.

En termes incrémentaux, nous définissons l'ensemble des incréments de traction admissibles $\Delta K_{\mu}$ comme suit :

$\Delta K_{\mu}=\left\{\left(\Delta t_{\mathrm{n}}, \Delta t_{\mathrm{t}}\right)\right.$ tel que $\left.\left\|t_{\mathrm{t} 0}+\Delta t_{\mathrm{t}}\right\| \leq \mu\left(t_{\mathrm{n} 0}+\Delta t_{\mathrm{n}}\right)\right\}$

La loi de contact et son inverse (6) deviennent respectivement :

$$
\left\{\begin{array}{l}
-\left(u_{1}-u_{0}\right)=-\Delta u \in \Delta \tau \partial_{\mathrm{t}} b_{\mathrm{c}}\left(\frac{-\Delta u}{\Delta \tau}, t_{0}+\Delta t\right) \\
t_{1}-t_{0}=\Delta t \in \Delta \tau \partial_{-\dot{u}} b_{\mathrm{c}}\left(\frac{-\Delta u}{\Delta \tau}, t_{0}+\Delta t\right)-t_{0}
\end{array}\right.
$$

De manière similaire au comportement plastique, la formulation du contact avec frottement sec de Coulomb est basée sur l'introduction de la fonction incrémentale suivante :

$$
\begin{array}{r}
\Delta b_{\mathrm{c}}(-\Delta u, \Delta t)=\Delta \tau b_{\mathrm{c}}\left(\frac{-\Delta u}{\Delta \tau}, t_{0}+\Delta t\right)-t_{0} \Delta u \\
=t_{\mathrm{n} 0} \Delta u_{\mathrm{n}}+t_{\mathrm{t} 0} \Delta u_{\mathrm{t}}+\mu\left(t_{\mathrm{n} 0}+\Delta t_{\mathrm{n}}\right)\left\|\Delta u_{\mathrm{t}}\right\|
\end{array}
$$

Puisque $\Delta \tau>0$ et le bipotentiel incrémental $\Delta b_{\mathrm{c}}$ est séparément convexe, donc pour tout couple $(\Delta u, \Delta t)$, nous avons :

$$
\Delta b_{\mathrm{c}}(-\Delta u, \Delta t) \geq-\Delta \tau \frac{\Delta u}{\Delta \tau}\left(t_{0}+\Delta t\right)-t_{0} \Delta u
$$




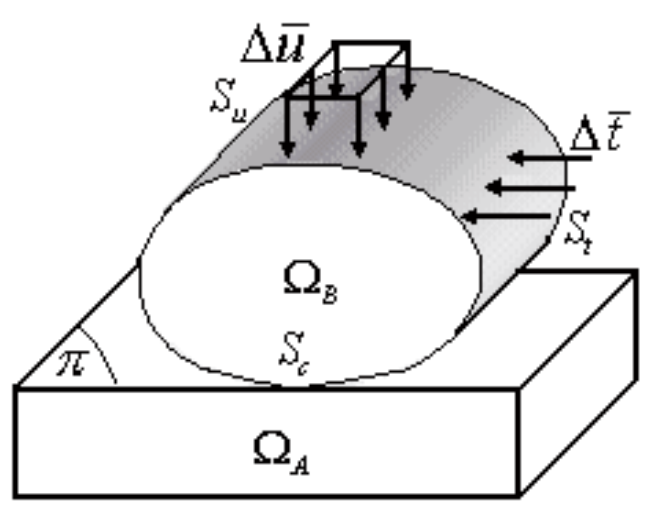

Fig. 3. Structure en 3D avec contact plus les conditions aux limites.

d'où

$$
\Delta b_{\mathrm{c}}(-\Delta u, \Delta t) \geq-\Delta t \Delta u
$$

Pour les applications numériques, les lois incrémentales de contact sont présentées sous la forme incrémentale suivante :

$$
\left\{\begin{array}{l}
-\Delta u \in \partial_{\Delta \mathrm{t}} b_{\mathrm{c}}(-\Delta u, \Delta t) \\
\Delta t \in \partial_{-\Delta \mathrm{u}} b_{\mathrm{c}}(-\Delta u, \Delta t)
\end{array}\right.
$$

En résumé, le schéma implicite nous a permis de calculer les éléments nécessaires qui sont le surpotentiel incrémental en élastoplasticité et le bipotentiel incrémental de contact dans le but d'établir la formulation variationnelle.

\section{Formulation variationnelle}

Soit une structure $\Omega_{\mathrm{B}}$, soumise à des forces de surface $\Delta \bar{t}$ sur la surface $S_{\mathrm{t}}$, à des forces de gravité $\Delta \bar{f}$ et des déplacements imposés $\Delta \bar{u}$ sur la surface $S_{\mathrm{u}}$. La partie $S_{\mathrm{c}}=S-S_{\mathrm{u}} \cup S_{\mathrm{t}}$ telle que $S_{\mathrm{c}} \cap S_{\mathrm{t}} \cap S_{\mathrm{u}}=\phi$ est une zone proposée d'être en contact avec $\Omega_{\mathrm{A}}$ au niveau du plan tangent commun $\pi$ comme l'indique la figure 3 .

Un champ des incréments de déplacements $\left(\Delta u^{\mathrm{c}}\right)$ est dit cinématiquement admissible, si les équations de compatibilité suivantes sont satisfaites :

$$
\left\{\begin{array}{l}
\Delta \varepsilon\left(\Delta u^{\mathrm{c}}\right)=\nabla_{\mathrm{s}} \Delta u^{\mathrm{c}} \text { dans } \Omega \\
\Delta u^{\mathrm{c}}=\Delta \bar{u} \text { sur } S_{\mathrm{u}}
\end{array}\right.
$$

Un champ des incréments de contraintes $\left(\Delta \sigma^{\mathrm{s}}\right)$ est dit statiquement admissible, si les équations d'équilibre suivantes sont satisfaites:

$$
\left\{\begin{array}{l}
\operatorname{div}\left(\Delta \sigma^{\mathrm{s}}\right)+\Delta \bar{f}=0 \text { dans } \Omega \\
\Delta t\left(\Delta \sigma^{\mathrm{s}}\right)=\Delta \sigma^{\mathrm{s}} n=\Delta \bar{t} \text { sur } S_{\mathrm{t}}
\end{array}\right.
$$

Un couple $(\Delta u, \Delta \sigma)$ est une solution du problème aux valeurs limites pour le contact unilatéral avec frottement sec de Coulomb et le matériau élastique parfaitement plastique, si ce couple satisfait les conditions suivantes :

$\left\{\begin{array}{l}\Delta u \text { est cinématiquement admissible, } \\ \Delta \sigma \text { est statiquement admissible, } \\ \text { le couple }(-\Delta u, \Delta t(\Delta \sigma)) \text { est extrémal pour } \Delta b_{\mathrm{c}} \operatorname{sur} S_{\mathrm{c}}, \\ \text { le couple }(-\Delta \varepsilon(\Delta u), \Delta \sigma) \text { est extrémal pour }(\Delta V+\Delta W) .\end{array}\right.$

Nous introduisons la fonction suivante, appelée bifonctionnelle :

$$
\begin{aligned}
& \Delta \beta(\Delta u, \Delta \sigma)=\int_{\Omega}[\Delta V(\Delta \varepsilon(u))-\Delta \bar{f} \Delta u] \mathrm{d} \Omega \\
& -\int_{S_{\mathrm{t}}} \Delta \bar{t} \Delta u \mathrm{~d} S-\int_{S_{\mathrm{u}}} \Delta t(\Delta \sigma) \Delta \bar{u} \mathrm{~d} S+\int_{S_{\mathrm{c}}} \Delta b_{\mathrm{c}}(-\Delta u, \Delta t) \mathrm{d} S
\end{aligned}
$$

Par cette définition, nous pouvons étendre le calcul classique des variations aux matériaux standards implicites. Nous prouvons que le champ des couples $(\Delta u, \Delta \sigma)$, solution exacte du problème aux valeurs limites, est simultanément solution des principes variationnels suivants :

$$
\left\{\begin{array}{c}
\operatorname{Inf}_{\Delta u^{\mathrm{c}} C \cdot A} \Delta \beta\left(\Delta u^{\mathrm{c}}, \Delta \sigma\right) \\
\underset{\Delta \sigma^{\mathrm{s}} S \cdot A}{\operatorname{Inf}} \Delta \beta\left(\Delta u, \Delta \sigma^{\mathrm{s}}\right)
\end{array}\right.
$$

Pour la formulation variationnelle en terme de déplacements, les termes qui ne dépendent pas du champ incrémental $\Delta u$ disparaissent et la bifonctionnelle $\Delta \beta\left(\Delta u^{\mathrm{c}}, \Delta \sigma\right)$ est réduite à :

$$
\begin{aligned}
& \Delta \Phi(\Delta u, \Delta \sigma)=\int_{\Omega}[\Delta V(\Delta \varepsilon(u))-\Delta \bar{f} \Delta u] \mathrm{d} \Omega \\
& -\int_{S_{\mathrm{t}}} \Delta \bar{t} \Delta u \mathrm{~d} S+\int_{S_{\mathrm{c}}} \Delta b_{\mathrm{c}}(-\Delta u, \Delta t(\Delta \sigma)) \mathrm{d} S
\end{aligned}
$$

Donc, le principe variationnel cinématique devient :

$$
\operatorname{Inf}_{\Delta u^{\mathrm{c} C} \cdot A} \Delta \Phi\left(\Delta u^{\mathrm{c}}, \Delta \sigma\right)
$$

\section{Discrétisation éléments-finis}

L'approximation du champ des incréments de déplacements est définie par les relations [15] :

$$
\left\{\begin{array}{l}
\Delta u(x)=N(x) \Delta U \\
\Delta \varepsilon=B \Delta U \text { avec } B=\operatorname{grad}_{\mathrm{s}}(N(x))
\end{array}\right.
$$

où $x=\langle x, y, z\rangle$ sont les coordonnées nodales, $\Delta U$ est le vecteur des incréments de déplacements nodaux inconnus et $N(x)$ est la matrice des fonctions de formes.

Introduisons le vecteur des incréments de forces généralisées :

$$
\Delta F=\int_{\Omega} N^{T} \Delta \bar{f} \mathrm{~d} \Omega+\int_{S_{\mathrm{t}}} N^{T} \Delta \bar{t} \mathrm{~d} S
$$


La bifonctionnelle (54) prend la forme discrétisée suivante :

$$
\begin{aligned}
\Delta \Phi(\Delta U)=\int_{\Omega} \Delta V(B \Delta U) \mathrm{d} \Omega-\Delta F & \\
& +\int_{S_{\mathrm{c}}} \Delta b_{\mathrm{c}}(-N \Delta U, \Delta t) \mathrm{d} S
\end{aligned}
$$

Dans ce cas, les incréments de contraintes sont non discrétisés comme les contraintes principales, mais peuvent être déduits à partir de la valeur des incréments de déplacements par la résolution du système d'équations :

$$
\left\{\begin{array}{l}
\int_{\Omega} B^{T} \Delta \sigma \mathrm{d} \Omega-\Delta F-\int_{S_{\mathrm{c}}} N^{T} \Delta t \mathrm{~d} S=0 \\
\Delta t \in \partial_{-\Delta u} \Delta b_{\mathrm{c}}(-N \Delta U, \Delta t) \\
\Delta \sigma=\frac{\partial \Delta V(B \Delta U)}{\partial \Delta \varepsilon}
\end{array}\right.
$$

\section{Algorithmes de résolution numérique}

\subsection{Algorithme de régularisation}

La régularisation de la loi de frottement de Coulomb consiste à contourner la non différentiabilité de celle-ci aux points où le glissement est nul.

Nous avons montré que le contact avec frottement peut être représenté par le bipotentiel suivant :

$$
\begin{array}{r}
\Delta b_{\mathrm{c}}(\Delta u, \Delta t)=t_{\mathrm{n} 0} \Delta u_{\mathrm{n}}+t_{\mathrm{t} 0} \Delta u_{\mathrm{t}}+\Delta t_{\mathrm{n}}\left(u_{\mathrm{n} 0}+h_{0}\right) \\
+\mu\left(t_{\mathrm{n} 0}+\Delta t_{\mathrm{n}}\right)\left\|\Delta u_{\mathrm{t}}\right\|
\end{array}
$$

Ce bipotentiel n'est pas partout différentiable ce qui pose des problèmes au niveau de la programmation mathématique.

Par analogie avec la résolution des problèmes de la plasticité, nous résolvons un problème de frottement similaire au comportement élastoplastique. Cela nécessite l'introduction d'une rigidité fictive $S_{c}^{-1}$. Nous pouvons écrire par la suite : $u=u^{\prime}+u^{\mathrm{f}}$ dont sa forme ressemble à celle de l'équation (7)) avec $u^{\prime}=S_{\mathrm{c}}^{-1} t$.

On obtient donc l'analogie suivante : $u^{\prime} \equiv \varepsilon^{\mathrm{e}}$ et $u^{\mathrm{f}} \equiv \varepsilon^{\mathrm{p}}$ comme l'indique la figure 4 .

Dans le cas de la traction unilatérale, nous avons :

$$
\varepsilon^{\mathrm{e}}=\frac{\sigma}{E} \text { quand } E \rightarrow \infty \varepsilon^{\mathrm{e}} \rightarrow 0
$$

Par le même raisonnement, nous posons :

$$
u^{\prime}=\frac{t}{k} \text { quand } k \rightarrow \infty u^{\prime} \rightarrow 0 \text { et } u \rightarrow u^{\mathrm{f}}
$$

Nous pouvons par la suite introduire le bipotentiel suivant :

$$
\Delta b^{\prime}=\frac{K_{\mathrm{t}}}{2}\left(-\Delta u_{\mathrm{t}}+\Delta u_{\mathrm{t}}^{\mathrm{f}}\right)^{2}+\frac{K_{\mathrm{n}}}{2}\left(-\Delta u_{\mathrm{n}}+\Delta u_{\mathrm{n}}^{\mathrm{f}}\right)^{2}
$$

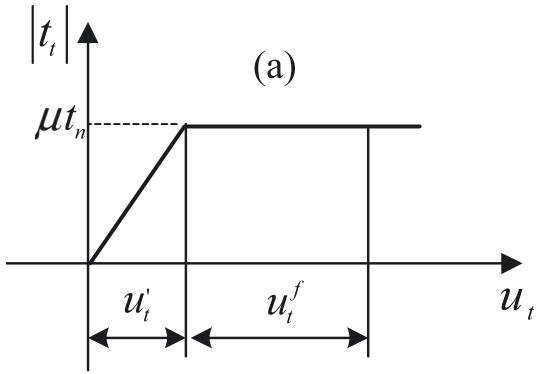

(b)

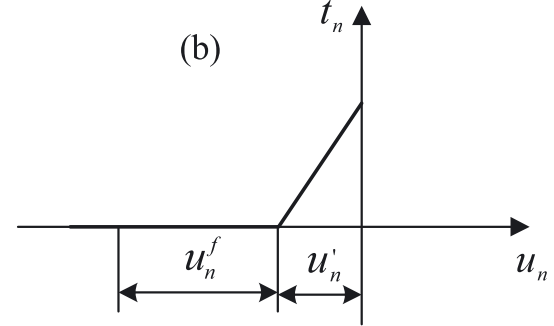

Fig. 4. Lois de frottement de Coulomb (a) régularisées et de contact (b).

qui sera intégré dans le bipotentiel incrémental $\Delta b_{\mathrm{c}}(\Delta u, \Delta t)$ pour faciliter sa minimisation. Les termes $K_{\mathrm{n}}$ et $K_{\mathrm{t}}$ sont des coefficients de pénalité.

Nous montrons que $\Delta b_{\mathrm{c}}(\Delta u, \Delta t)$ peut s'écrire comme suit :

$$
\Delta b_{\mathrm{c}}(\Delta u, \Delta t)=\Delta b_{\mathrm{n}}(\Delta u, \Delta t)+\Delta b_{\mathrm{t}}(\Delta u, \Delta t)
$$

avec

$$
\left\{\begin{array}{l}
\Delta b_{\mathrm{n}}=\operatorname{Inf}_{-\Delta u_{\mathrm{n}}^{\mathrm{f}}}\left\{-t_{\mathrm{n} 0}\left(-\Delta u_{\mathrm{n}}^{\mathrm{f}}\right)+\frac{K_{\mathrm{n}}}{2}\left(-\Delta u_{\mathrm{n}}+\Delta u_{\mathrm{n}}^{\mathrm{f}}\right)^{2}\right\} \\
\text { et } \Delta u_{\mathrm{n}}^{\mathrm{f}}+u_{\mathrm{n} 0}+h_{0} \geq 0 \\
\begin{array}{l}
\Delta b_{\mathrm{t}}=\operatorname{Inf}_{-\Delta u_{\mathrm{t}}^{\mathrm{f}}}\left\{-t_{\mathrm{t} 0}\left(-\Delta u_{\mathrm{t}}^{\mathrm{f}}\right)+\mu\left(t_{\mathrm{n} 0}+\Delta t_{\mathrm{n}}\right)\left\|-\Delta u_{\mathrm{t}}^{\mathrm{f}}\right\|\right. \\
\left.+\frac{K_{\mathrm{t}}}{2}\left(-\Delta u_{\mathrm{t}}+\Delta u_{\mathrm{t}}^{\mathrm{f}}\right)^{2}\right\}
\end{array}
\end{array}\right.
$$

\subsubsection{Problème de contact seul}

Nous reprenons l'équation (65a) et d'après la condition de stationnarité, nous avons :

$$
\frac{\partial \Delta b_{\mathrm{n}}(\Delta u, \Delta t)}{\partial\left(-\Delta u_{\mathrm{n}}^{\mathrm{f}}\right)}=0 \Rightarrow \Delta u_{\mathrm{n}}^{\mathrm{f}}=-\frac{t_{\mathrm{n} 0}}{K_{\mathrm{n}}}+\Delta u_{\mathrm{n}}
$$

Si nous posons : $c=\Delta u_{\mathrm{n}}^{\mathrm{f}}+x_{0}$ avec $x_{0}=u_{\mathrm{n} 0}+h_{0}$

La condition de non contact $(c>0)$ nous donne :

$$
\Delta u_{\mathrm{n}}^{\mathrm{f}}=-\frac{t_{\mathrm{n} 0}}{K_{\mathrm{n}}}+\Delta u_{\mathrm{n}}
$$

Par substitution de (67) dans (65a), elle devient :

$$
\begin{aligned}
\Delta b_{\mathrm{n}} & =-t_{\mathrm{n} 0}\left(-\Delta u_{\mathrm{n}}+\frac{t_{\mathrm{n} 0}}{K_{\mathrm{n}}}\right)+\frac{K_{\mathrm{n}}}{2}\left(-\Delta u_{\mathrm{n}}+\Delta u_{\mathrm{n}}-\frac{t_{\mathrm{n} 0}}{K_{\mathrm{n}}}\right)^{2} \\
& =t_{\mathrm{n} 0} \Delta u_{\mathrm{n}}-\frac{t_{\mathrm{n} 0}^{2}}{2 K_{\mathrm{n}}}
\end{aligned}
$$


De l'équation (68), nous déduisons la valeur de l'incrément de contrainte normale :

$$
\Delta t_{\mathrm{n}}=\frac{\partial \Delta b_{\mathrm{n}}(-\Delta u, \Delta t)}{\partial\left(-\Delta u_{\mathrm{n}}\right)}=-t_{\mathrm{n} 0}
$$

La condition $d u$ contact est réalisée quand le paramètre $c \leq 0$, dans ce cas : $\Delta u_{\mathrm{n}}^{\mathrm{f}}=x_{0}$ puis,

$$
\begin{gathered}
\Delta b_{\mathrm{n}}(u, t)=-t_{\mathrm{n} 0} x_{0}+\frac{K_{\mathrm{n}}}{2}\left(\Delta u_{\mathrm{n}}+x_{0}\right)^{2} \Rightarrow \\
\Delta t_{\mathrm{n}}=-K_{\mathrm{n}}\left(\Delta u_{\mathrm{n}}+x_{0}\right)
\end{gathered}
$$

\subsubsection{Condition de stationnarité}

La condition de stationnarité de $\left(\Delta b_{\mathrm{t}}\right)$, par rapport à $\left(-\Delta u_{\mathrm{t}}\right)$, est exprimée par la relation suivante :

$$
\frac{\partial \Delta b_{\mathrm{t}}(\Delta u, \Delta t)}{\partial\left(-\Delta u_{\mathrm{t}}^{\mathrm{f}}\right)}=0
$$

À partir des relations (69) et (71), nous obtenons

$$
\left(K_{\mathrm{t}}\left\|\Delta u_{\mathrm{t}}^{\mathrm{f}}\right\|+\mu\left(t_{\mathrm{n} 0}+\Delta t_{\mathrm{n}}\right)\right)\left(\frac{-\Delta u_{\mathrm{t}}^{\mathrm{f}}}{\left\|\Delta u_{\mathrm{t}}^{\mathrm{f}}\right\|}\right)=t_{\mathrm{t} 0}-K_{\mathrm{t}} \Delta u_{\mathrm{t}}
$$

et puisque le vecteur normal est égal à :

$$
n=\frac{-\Delta u_{\mathrm{t}}^{\mathrm{f}}}{\left\|-\Delta u_{\mathrm{t}}^{\mathrm{f}}\right\|}=\frac{-\Delta u_{\mathrm{t}}+t_{\mathrm{t} 0} / K_{\mathrm{t}}}{\left\|-\Delta u_{\mathrm{t}}+t_{\mathrm{t} 0} / K_{\mathrm{t}}\right\|}
$$

d'où $\left(K_{\mathrm{t}}\left\|\Delta u_{\mathrm{t}}^{\mathrm{f}}\right\|+\mu\left(t_{\mathrm{n} 0}+\Delta t_{\mathrm{n}}\right)\right)=K_{\mathrm{t}}\left(\frac{t_{\mathrm{t} 0}}{K_{\mathrm{t}}}-\Delta u_{\mathrm{t} 0}\right)$ $\times \frac{\left\|-\Delta u_{\mathrm{t}}+t_{\mathrm{to} 0} / K_{\mathrm{t}}\right\|}{-\Delta u_{\mathrm{t}}+t_{\mathrm{t} 0} / K_{\mathrm{t}}}$

En calculant la norme des deux membres de l'équation (72), nous obtenons

$$
a=\left\|\Delta u_{\mathrm{t}}^{\mathrm{f}}\right\|=\left[\left\|-\Delta u_{\mathrm{t}}+t_{\mathrm{t} 0} / K_{\mathrm{t}}\right\|-\frac{\mu}{K_{\mathrm{t}}}\left(t_{\mathrm{n} 0}+\Delta t_{\mathrm{n}}\right)\right]_{+}
$$

Si $a>0$, alors nous avons :

$$
\begin{aligned}
& \Delta u_{\mathrm{t}}^{\mathrm{f}}=-a n \text { et } \Delta b_{\mathrm{t}}=\frac{K_{\mathrm{t}}}{2}\left(-\Delta u_{\mathrm{t}}+\Delta u_{\mathrm{t}}^{\mathrm{f}}\right)^{2} \\
& +t_{\mathrm{t} 0} \Delta u_{\mathrm{t}}^{\mathrm{f}}+\mu\left(t_{\mathrm{n} 0}+\Delta t_{\mathrm{n}}\right)\left\|\Delta u_{\mathrm{t}}^{\mathrm{f}}\right\|
\end{aligned}
$$

L'incrément de la contrainte tangentielle est donné par :

$$
\begin{aligned}
\Delta t_{\mathrm{t}}=\frac{\partial \Delta b_{\mathrm{t}}(u, t)}{\partial\left(-\Delta u_{\mathrm{t}}\right)}= & K_{\mathrm{t}}\left(-\Delta u_{\mathrm{t}}+\Delta u_{\mathrm{t}}^{\mathrm{f}}\right)\left(1+\frac{\partial \Delta u_{\mathrm{t}}^{\mathrm{f}}}{\partial\left(-\Delta u_{\mathrm{t}}\right)}\right. \\
& +t_{\mathrm{t} 0} \frac{\partial \Delta u_{\mathrm{t}}^{\mathrm{f}}}{\partial\left(-\Delta u_{\mathrm{t}}\right)}+\mu\left(t_{\mathrm{n} 0}+\Delta t_{\mathrm{n}}\right) \frac{\partial\left\|\Delta u_{\mathrm{t}}^{\mathrm{f}}\right\|}{\partial\left(-\Delta u_{\mathrm{t}}\right)}
\end{aligned}
$$

Or $\frac{\partial n}{\partial\left(-\Delta u_{\mathrm{t}}\right)}=0$ et $\Delta u_{\mathrm{t}}^{\mathrm{f}}=\frac{\Delta u_{\mathrm{t}}^{\mathrm{f}}}{\left\|\Delta u_{\mathrm{t}}^{\mathrm{f}}\right\|} \cdot\left\|\Delta u_{\mathrm{t}}^{\mathrm{f}}\right\|=-n\left\|\Delta u_{\mathrm{t}}^{\mathrm{f}}\right\|$. Puis $\frac{\partial \Delta u_{\mathrm{t}}^{\mathrm{f}}}{\partial\left(-\Delta u_{\mathrm{t}}\right)}=-1$ et $\frac{\partial\left\|\Delta u_{\mathrm{tt}}^{\mathrm{f}}\right\|}{\partial\left(-\Delta u_{\mathrm{t}}\right)}=n$

D'où, l'incrément de la contrainte tangentielle est exprimé par :

$$
\Delta t_{\mathrm{t}}=\mu\left(t_{\mathrm{n} 0}+\Delta t_{\mathrm{n}}\right) n-t_{\mathrm{t} 0}
$$

\subsection{Méthode de lagrangien augmenté avec algorithme d'Uzawa}

Afin de surmonter la non différentiabilité du bipotentiel du contact frottant, nous adoptons la technique du lagrangien augmenté avec algorithme d'Uzawa. Ceci conduit à un schéma itératif de prédiction/correction permettant la détermination de la valeur de la réaction de contact $\Delta t$.

Dans le cas du contact, nous obtenons l'équation suivante :

$$
\begin{aligned}
\Delta t=\operatorname{proj}\left(\Delta t_{\mathrm{n}}-\rho\left(\Delta u_{\mathrm{n}}+u_{\mathrm{n} 0}+h_{0}\right.\right. & \left.+\mu\left\|\Delta u_{\mathrm{t}}\right\|\right), \Delta t_{\mathrm{t}} \\
& \left.-\rho \Delta u_{\mathrm{t}}, \Delta K_{\mu}\right)
\end{aligned}
$$

où $\rho$ est un coefficient positif dont la valeur doit être bien choisie pour assurer la convergence numérique.

L'application de l'algorithme de projection d'Uzawa conduit à un schéma itératif composé de deux étapes :

Prédiction :

$$
\left\{\begin{array}{l}
\Delta \hat{t}_{\mathrm{n}}^{i+1}=\Delta t_{\mathrm{n}}^{i}-\rho\left(\Delta u_{\mathrm{n}}\left(\Delta t_{\mathrm{n}}^{i}, \Delta t_{\mathrm{t}}^{i}\right)+u_{\mathrm{n} 0}+h_{0}+\mu\right. \\
\left.\quad \times\left\|\Delta u_{\mathrm{t}}\left(\Delta t_{\mathrm{n}}^{i}, \Delta t_{\mathrm{t}}^{i}\right)\right\|\right) \\
\Delta \hat{t}_{\mathrm{t}}^{i+1}=\Delta t_{\mathrm{t}}^{i}-\rho \Delta u_{\mathrm{t}}\left(\Delta t_{\mathrm{n}}^{i}, \Delta t_{\mathrm{t}}^{i}\right)
\end{array}\right.
$$

\section{Correction :}

$$
\Delta t^{i+1}=\operatorname{proj}\left(\Delta \hat{t}^{i+1}, \Delta K_{\mu}\right)
$$

où $\Delta \hat{t}^{i+1}$ désigne la prédiction de la réaction de contact à l'itération $i+1$.

Cet algorithme peut être présenté comme suit :

$\left\{\begin{array}{l}\operatorname{si} \mu\left|\Delta \hat{t}_{\mathrm{t}}^{i+1}\right|<-\Delta \hat{t}_{\mathrm{n}}^{i+1} \text { alors } \Delta t^{i+1}=0 \text { pas de contact } \\ \text { sinon si }\left|\Delta \hat{t}_{\mathrm{t}}^{i+1}\right|<\mu \Delta \hat{t}_{\mathrm{n}}^{i+1} \text { alors } \Delta t^{i+1}=\Delta \hat{t}^{i+1} \text { adhérence } \\ \text { sinon } \Delta t^{i+1}=\Delta \hat{t}^{i+1}-\frac{\left(\left|\Delta \hat{t}_{\mathrm{t}}^{i+1}\right|-\mu \Delta \hat{t}_{\mathrm{n}}^{i+1}\right)}{1+\mu^{2}}\left(\frac{\Delta \hat{t}_{\mathrm{t}}^{i+1}}{\left|\Delta \hat{t}_{\mathrm{t}}^{i+1}\right|}+\mu n\right) \\ \quad \text { glissement }\end{array}\right.$

d'où

$$
\left\{\begin{array}{l}
\Delta t_{\mathrm{t}}^{i+1}=\Delta \widetilde{t}_{\mathrm{t}}^{i+1}-\frac{\left(\left|\Delta \widehat{t}_{\mathrm{t}}^{i+1}\right|-\mu \Delta \widehat{t}_{\mathrm{n}}^{i+1}\right)}{1+\mu^{2}} \\
\Delta t_{\mathrm{n}}^{i+1}=\Delta \bar{t}_{\mathrm{n}}^{i+1}-\frac{\left(\left|\Delta \widehat{t}_{\mathrm{t}}^{i+1}\right|-\mu \Delta \widehat{t}_{\mathrm{n}}^{i+1}\right)}{1+\mu^{2}} \mu
\end{array}\right.
$$

\subsection{Méthode du point fixe}

Le couple $(-\Delta u, \Delta \sigma)$, solution du problème aux limites, est à l'intersection de l'hypersurface $(\Gamma)$ définie par les lois constitutives $(59 \mathrm{~b}, 59 \mathrm{c})$ et du sous espace affine (EA) des solutions cinématiquement admissibles, défini par l'équation (59a), (voir Fig. 5).

La résolution de $(59 \mathrm{~b}, 59 \mathrm{c})$ correspond à l'étape locale, représentée par la direction de montée $\mathrm{E}^{+}$, et la résolution de $(59 a)$ correspond à l'étape globale, représentée par la direction de descente $\mathrm{E}^{-}$. 


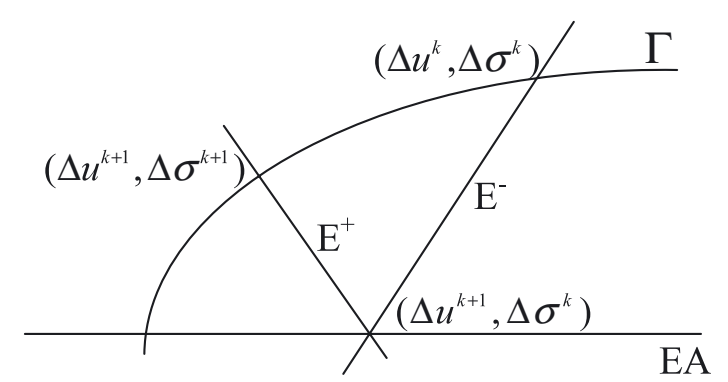

Fig. 5. Schéma de la méthode du point fixe.

\subsection{1 Étape globale}

$\Delta \sigma^{k}$ est fixé et une nouvelle approximation de $\Delta u^{k+1}$ est calculée par la minimisation de (59a) à l'aide du code de programmation mathématique Minos [5]. Le problème des déplacements consiste à minimiser l'équation (58). Pour accélérer la procédure d'optimisation, l'estimation $\left(\Delta U^{k}, \Delta t^{k}\right)$ est donnée comme point initial.

\subsection{2 Étape locale}

$\Delta U^{k+1}$ est fixé et une nouvelle approximation de l'incrément de la réaction $\Delta t^{k+1}$ est calculée :

$$
\Delta t^{k+1}=\frac{\partial \Delta b_{\mathrm{c}}\left(B \Delta U^{k+1}, \Delta t^{k}\right)}{\partial(-\Delta u)}
$$

Le critère d'arrêt des itérations est basé sur l'erreur relative aux lois constitutives implicites. L'estimation de l'erreur suivante est considérée :

$$
\begin{aligned}
& \varepsilon_{\text {err }}= \\
& \frac{\left(\int_{\Omega}\left\|\Delta \sigma^{k+1}-\Delta \sigma^{k}\right\|^{2} \mathrm{~d} \Omega+\int_{S_{\mathrm{c}}}\left\|\Delta t^{k+1}-\Delta t^{k}\right\|^{2} \mathrm{~d} S\right)^{1 / 2}}{\left(\int_{\Omega}\left\|\Delta \sigma^{k}\right\|^{2} \mathrm{~d} \Omega+\int_{S_{\mathrm{c}}}\left\|\Delta t^{k}\right\|^{2} \mathrm{~d} S\right)^{1 / 2}} \\
& \leq \varepsilon_{\text {tol }}
\end{aligned}
$$

où $\varepsilon_{\text {tol }}$ est la valeur de tolérance qui dépend de la précision cherchée et du coût de calcul, par expérience, nous prenons $10^{-3}$ à $10^{-6}$.

N.B. : l'organigramme général du processus de résolution est donné en annexe (voir Fig. 20).

\section{Applications numériques}

\subsection{Exemple de validation}

Le but de cet exemple est la validation de nos résultats par comparaison avec ceux de Feng [4]. Dans ce cadre, nous considérons le contact avec frottement entre un bloc élastique et une fondation rigide (Fig. 6). Le bloc est en état plan de déformation, supporte une charge $F=$ $1000 \mathrm{~N}$, son matériau a un module de Young $E=$ $210000 \mathrm{MPa}$ et un coefficient de Poisson $\nu=0,3$ et ses

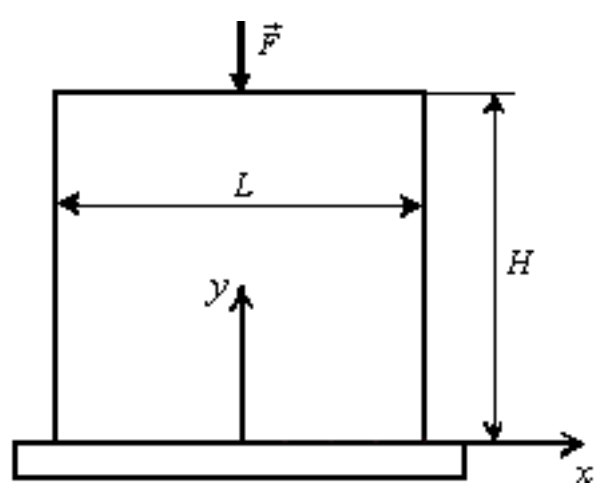

Fig. 6. Géométrie et conditions aux limites.

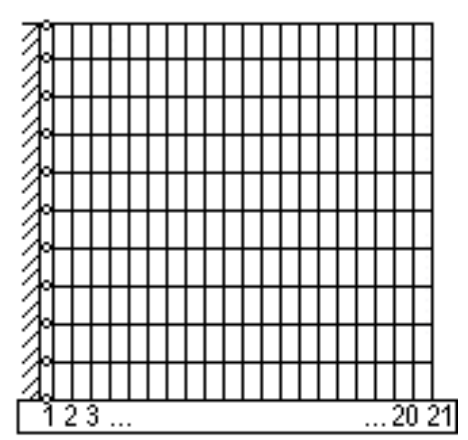

Fig. 7. Maillage de la moitié du bloc (200 éléments quadratiques à 4 nouds).

dimensions sont $L \times H=4 \times 2 \mathrm{~mm}^{2}$. Le coefficient de frottement entre le bloc et la fondation vaut $\mu=0,6$.

Tenant compte de la symétrie du bloc, on ne modélise que sa moitié $x \times y=2 \times 2$, (voir Fig. 7).

Les valeurs des erreurs relatives des forces normales et tangentielles de contact, au nœud remarquable d'abscisse $0,8 \mathrm{~mm}$, sont données respectivement sous forme de tableaux 1 et 2 .

Notons que :

$$
\begin{aligned}
\text { Erreur relative }= & \frac{\mid \text { Résultat }_{\text {Algorithme présent }}}{\text { Résultat }_{\text {Algorithme de Feng }}} \\
& -\frac{\text { Résultat }_{\text {Algorithme de Feng }} \mid}{\text { Résultat }_{\text {Algorithme de Feng }}} \times 100 \%
\end{aligned}
$$

Nous pouvons noter que les valeurs des erreurs relatives correspondantes aux résultats calculés par les deux algorithmes sont petites.

Ainsi, les courbes ci-dessus des figures 8 et 9 montrent que les deux méthodes adoptées pour la résolution des problèmes élastoplastiques en présence du contact avec frottement sec de Coulomb, donnent des résultats très proches.

\subsection{Poinçonnement d'une tôle épaisse}

Une tôle épaisse carrée, simplement appuyée sur la face inférieure, vient en contact en son centre avec 
Tableau 1. Valeurs des erreurs relatives correspondantes aux forces normales de contact.

\begin{tabular}{lcc}
\hline Algorithmes & Forces nodales normales (MPa) & Erreurs relatives (\%) \\
\hline Uzawa et Minos (présent) & 280 & 1,08 \\
Régularisation et Minos (présent) & 268 & 3,2 \\
Uzawa et Newton-Raphson [Feng] & 277 & $* * * * * * *$ \\
\hline
\end{tabular}

Tableau 2. Valeurs des erreurs relatives correspondantes aux forces tangentielles de contact.

\begin{tabular}{lcc}
\hline Algorithmes & Forces nodales tangentielles (MPa) & Erreurs relatives (\%) \\
\hline Uzawa et Minos (présent) & -146 & 6,41 \\
Régularisation et Minos (présent) & -150 & 3,8 \\
Uzawa et Newton-Raphson [Feng] & -156 & $* * * * * * *$ \\
\hline
\end{tabular}

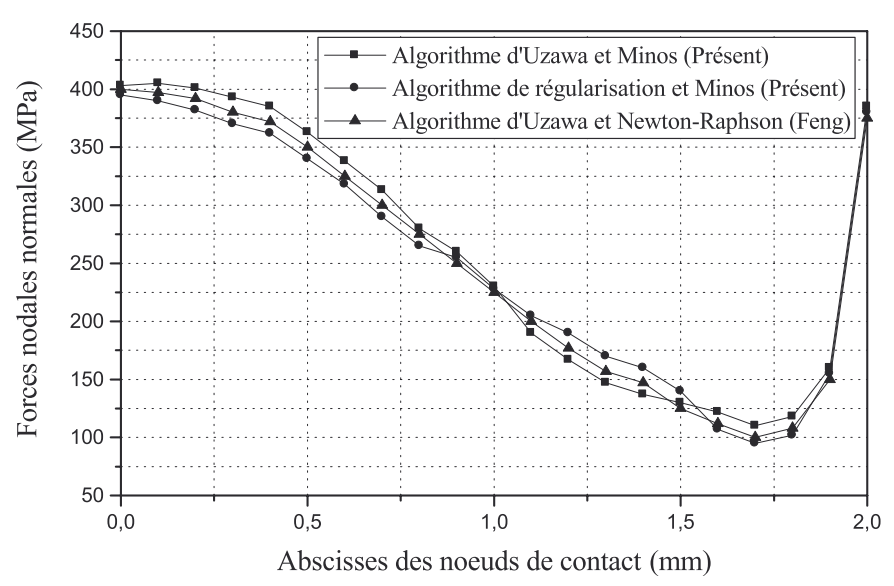

Fig. 8. Distribution des forces nodales normales.

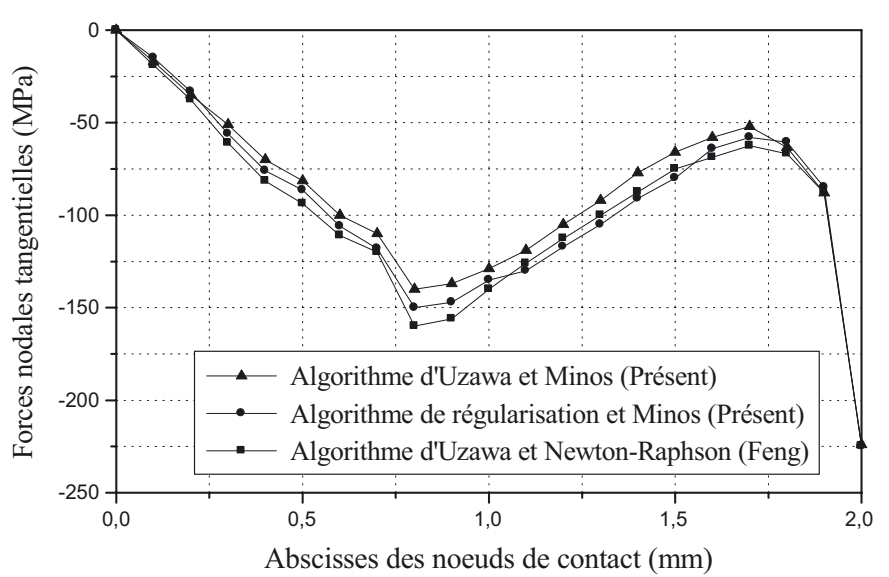

Fig. 9. Distribution des forces nodales tangentielles.

un poinçon parallélépipède [16-19]. La géométrie et le maillage sont illustrés par les figures 10 à 12 . Nous imposons un déplacement vertical constant $\left(U_{z}=-0,01 \mathrm{~mm}\right)$ sur la face supérieure du poinçon qui est considéré rigide, les deux pièces étant initialement en contact.

Le matériau est supposé élastique parfaitement plastique, obéissant au critère de von-Mises. Ses propriétés mécaniques sont : problème en $3 D$, limite élastique $\left(\sigma_{Y}=\right.$ $300 \mathrm{MPa})$, module de Young $\left(E=10^{5} \mathrm{MPa}\right)$, coefficient

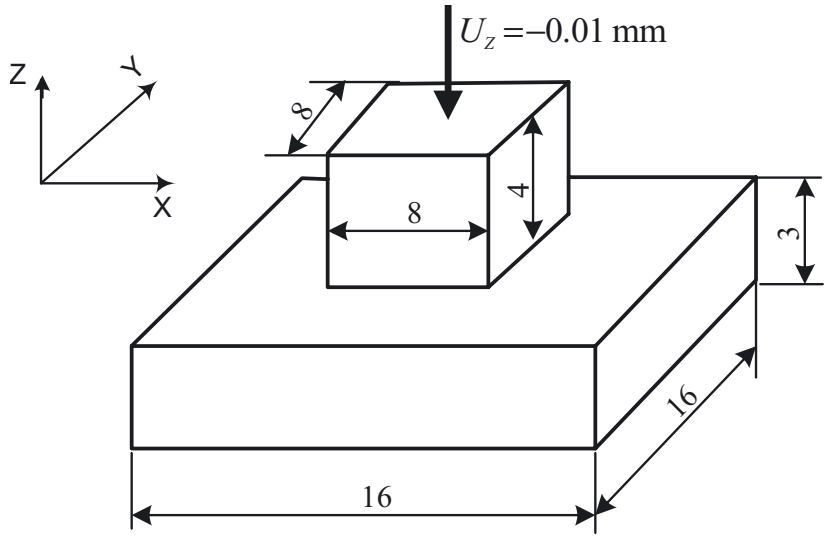

Fig. 10. Géométrie de la structure en 3D avec contact.
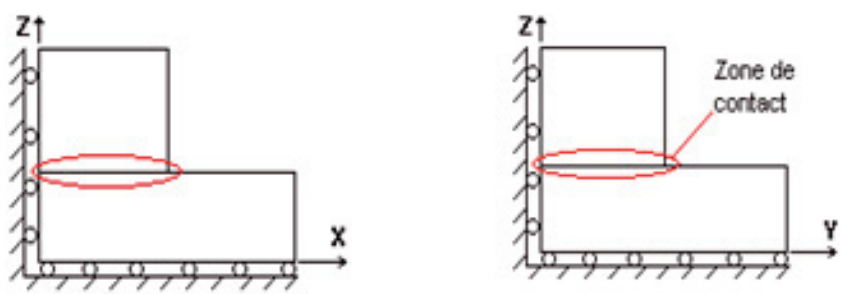

Fig. 11. Conditions aux limites dans les plans $(X Z)$ et $(Y Z)$.

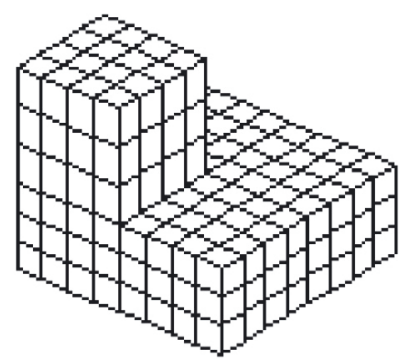

Fig. 12. Maillage (256 éléments hexaédriques à 8 nœuds).

de Poisson $(\nu=0,3)$ et coefficient de frottement $(\mu=0,1)$.

Tenant compte de la symétrie de la pièce à poinçonner, nous ne modélisons que le quart de la structure dont les dimensions sont $x \times y \times z=8 \times 8 \times 3 \mathrm{~mm}^{3}$. 

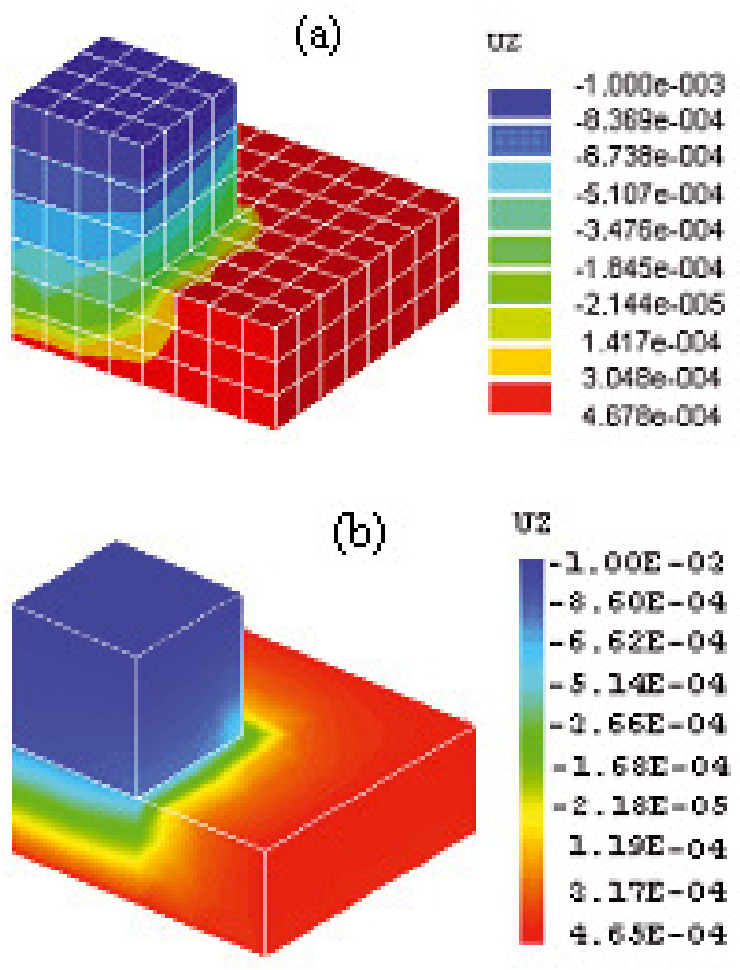

Fig. 13. Déformée de la structure obtenue par notre algorithme (a) et par CAST3M [21] (b).

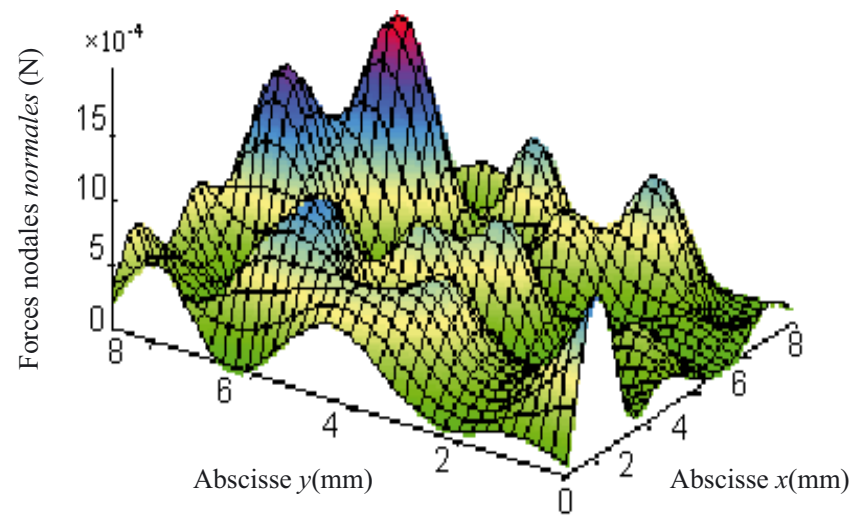

Fig. 14. Distribution des forces nodales normales aux nœuds du contact.

À partir des figures 14 à 16 , nous remarquons une concentration de contraintes et un glissement relatif maximal aux bords et surtout au coin de la surface de contact.

\subsection{Compression d'un bloc cylindrique}

Dans cet exemple, le bloc a une dimension de $10 \mathrm{~mm}$ de diamètre et de $10 \mathrm{~mm}$ de haut. Il est comprimé par deux plaques rigides (Fig. 17). Le matériau est supposé élastoplastique avec écrouissage isotrope, obéissant au

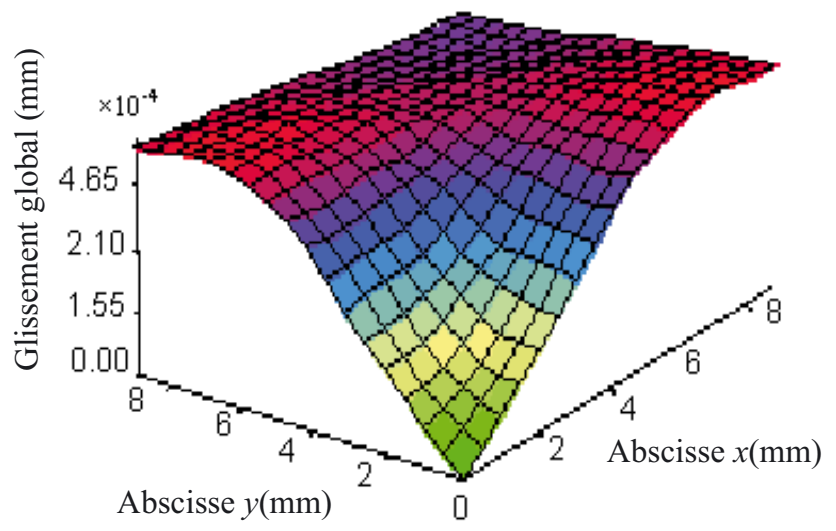

Fig. 15. Distribution du glissement aux nœuds du contact.

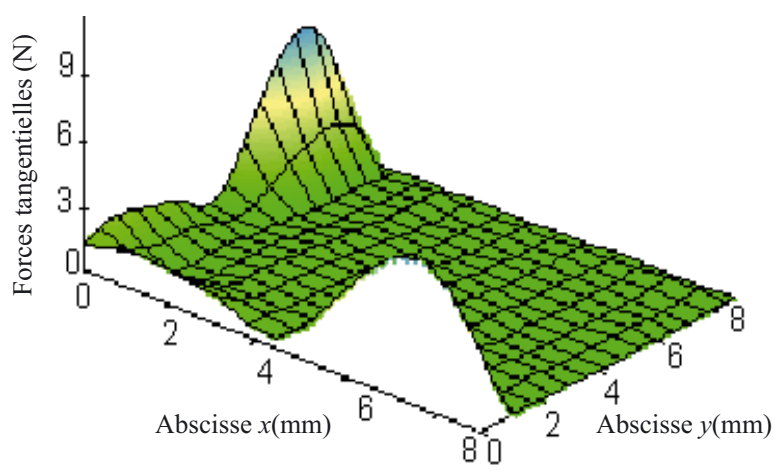

Fig. 16. Distribution des forces nodales tangentielles aux nœuds du contact.

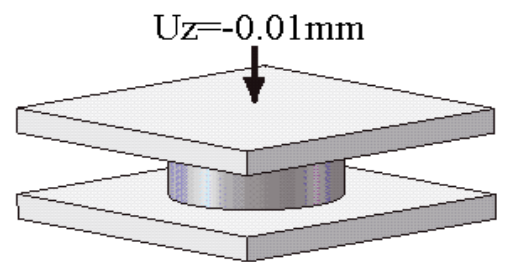

Fig. 17. Conditions aux limites du bloc cylindrique.

critère de von Mises [20]. La fonction de charge est définie par :

$$
f\left(\sigma, \bar{\varepsilon}^{\mathrm{p}}\right)=\left(\frac{3}{2} \sigma_{i j}^{\prime} \sigma_{i j}^{\prime}\right)^{0,5}-\sigma_{Y}\left(\bar{\varepsilon}^{\mathrm{p}}\right)
$$

où $\sigma_{i j}^{\prime}$ est la composante du tenseur déviateur des contraintes, $\sigma_{Y}$ dénote la contrainte d'écoulement et $\bar{\varepsilon}^{\mathrm{p}}$ indique la déformation plastique effective et son taux de déformation équivalente est défini par :

$$
\dot{\bar{\varepsilon}}^{\mathrm{p}}=\left(\frac{2}{3} \dot{\varepsilon}_{i j}^{\mathrm{p}} \dot{\varepsilon}_{i j}^{\mathrm{p}}\right)^{0,5}
$$

où le taux de déformation plastique $\dot{\varepsilon}^{\mathrm{p}}$ est défini dans l'équation (8).

La règle d'écrouissage linéaire est représentée comme suit :

$$
\sigma_{Y}=Y_{0}+h \bar{\varepsilon}^{\mathrm{p}}
$$


Tableau 3. Valeurs des erreurs absolues à $25 \%$ et $45 \%$ de réduction de hauteur.

\begin{tabular}{|l|l|c|c|}
\cline { 3 - 4 } \multicolumn{2}{c|}{} & \multicolumn{2}{c|}{ Déplacements $(\mathrm{mm})$} \\
\cline { 3 - 4 } \multicolumn{2}{c|}{ Algorithmes } & $25 \%$ & $45 \%$ \\
\cline { 2 - 4 } & Uzawa et Minos & 1,498799 & 3,422018 \\
\hline & Régularisation et Minos & 1,568904 & 3,402909 \\
\hline Erreurs absolues (mm) & 0,070105 & 0,019109 \\
\hline
\end{tabular}

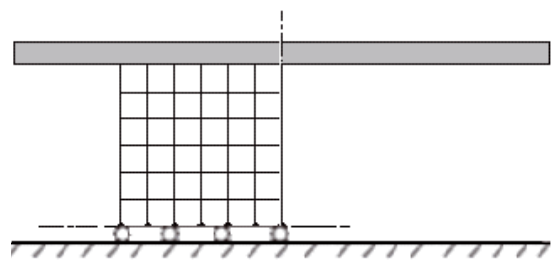

Fig. 18. Discrétisation axisymétrique du bloc (36 éléments quadrilatéraux à 4 nœuds).

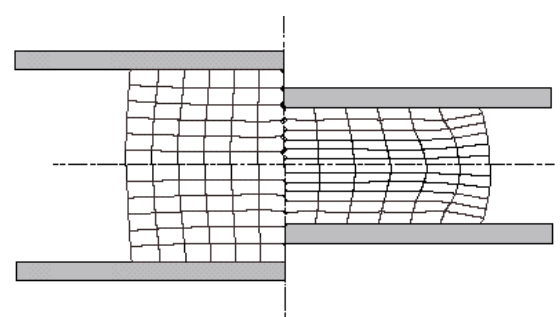

Fig. 19. Déformée du bloc obtenue à $25 \%$ (moitié gauche) et $45 \%$ (moitié droite) de réduction par les deux algorithmes : Uzawa-Minos (moitié en haut) et RégularisationMinos (moitié en bas).

où $Y_{0}$ est la contrainte d'écoulement initial, $h$ le coefficient d'écrouissage linéaire.

Les propriétés mécaniques du bloc sont : problème axisymétrique, limite élastique $\left(\sigma_{Y}=32 \mathrm{MPa}\right)$, module de Young $\left(E=375 \times 10^{2} \mathrm{MPa}\right)$, coefficient de Poisson $(\nu=0,25)$ et coefficient de frottement $(\mu=0,2)$.

À cause de la symétrie de la géométrie, seulement le quart du bloc est modélisé (Fig. 18).

La figure 19 présente l'accord entre les profils prévus par les deux algorithmes : Uzawa-Minos (moitié en haut) et Régularisation-Minos (moitié en bas). Les valeurs des erreurs absolues à $25 \%$ et $45 \%$ de réduction de hauteur, sont données sous forme de tableaux 3 .

Avec

Erreur absolue $=\mid$ Résultat $_{\text {Uzawa }}-$ Résultat $_{\text {Régularisation }} \mid$

Nous remarquons que les valeurs des erreurs absolues correspondantes aux résultats calculés par les deux algorithmes sont petites. En effet, la valeur de l'erreur absolue est $0,070105 \mathrm{~mm}$ à la réduction de $25 \%$ de hauteur et elle est seulement $0,019109 \mathrm{~mm}$ à la réduction de $45 \%$ de hauteur.

\section{Conclusion}

En conclusion, dire que la maîtrise des conditions de contact et de frottement est l'un des éléments essentiels du succès des opérations de mise en forme par déformation peut apparaître comme un euphémisme : si l'on songe qu'un produit pour arriver à sa forme finale, a suivi plusieurs dizaines de séquences de mise en forme et qu'à chaque séquence, ou presque, les conditions surfaciques changent, il est aisé d'imaginer les difficultés techniques et de situer l'énorme incidence économique qui correspond.

En effet, les approches proposées présentent un ajout dans le cadre de la simulation numérique des procédés de mise en forme, pour une bonne prise en compte des conditions aux limites dans les zones de contact. Les résultats obtenus sont globalement satisfaisants même si les surfaces de contact des exemples traités sont simples. Ceci montre l'efficacité des algorithmes adoptés et la fiabilité du programme développé.

Pour poursuivre ce travail, nous envisagerons la modélisation et simulation numérique du contact frottant avec échange thermique entre corps déformables dans le cas des grandes déformations.

\section{Annexe}

L'organigramme général du processus de résolution numérique est representé figure 20.

\section{Références}

[1] R. Hill, The mathematical theory of plasticity, Clarendon Press, Oxford, 1950

[2] J.J. Moreau, La notion de surpotentiel et les liaisons unilatérales en élastoplasticité, C.R. Acad. Sci. Paris, t. 267, 1968

[3] G. De Saxcé, Une généralisation de l'inégalité de Fenchel et ses applications aux lois constitutives, CR Acad. Sci. Paris, t. 314, 1992, pp. 125-129

[4] G. De Saxcé, Z.Q. Feng, The bipotential method: a constructive approach to design the complete contact law friction and improved numerical algorithms, Math. Comput. Model. 28 (1998) 4-8

[5] A. Murtagh and Saunders, Minos 5.1 user's guide, Standford Univesity, 1987

[6] M. Minoux, Programmation mathématique, Vol. 1, Dunod, 1983 


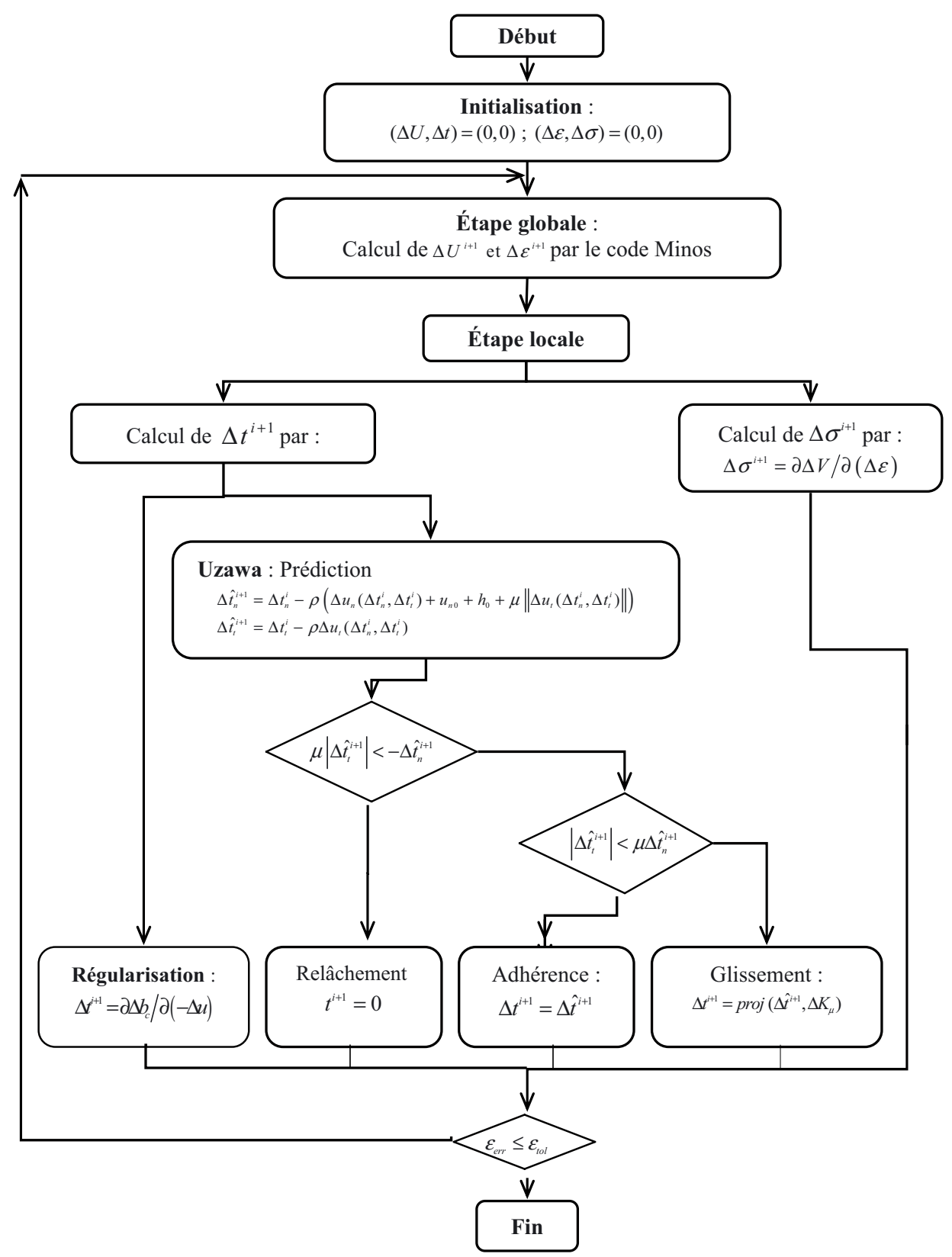

Fig. 20. Organigramme général du processus de résolution.

[7] M.R. Hestness, Multiplier and gradient methods, J. Optimiz. Theory Appl. Mech. 4 (1969) 303-320

[8] M.J.D. Powell, A Method for nonlinear constraints in minimization problems, in optimization, R. Fletcher (ed.), Acad. Press, New York, 1969, pp. 283-298

[9] A. Klarbring, Mathematical programming in contact problems, in computational methods in contact mechanics, Computational Mechanics Publications, Southampton, 1992, pp. 233-263

[10] G. De Saxcé, Z.Q. Feng, New inequality and functional for contact with friction: the implicite standard material approach, Mech. Struct. Math. 19 (1991) 301-325

[11] M. Jean, G. Touzot, Implementation of unilateral contact and dry friction in computer codes dealing with large deformation problems, J. Theor. Appl. Mech., special issue, supplement, Vol. 7 (1988)
[12] N. Kikuchi, J.T. Oden, Contact problems in elasticity a study of variational inequalities and finite element methods, Society for Industrial and Applied Mathematics, 1988

[13] J.J. Moreau, Proximité et dualité dans un espace Hilbertien, Bull. Soc. Math., France 93 (1965) 273-299

[14] Z.Q. Feng, G. De Saxcé, Rigid-plastic implicit integration scheme for analysis of metal forming, Eur. J. Mech. A/Solids 15 (1996) 51-66

[15] G. Dhatt, G. Touzot, Une présentation de la méthode des éléments finis, Maloine S.A, 1984

[16] E. Boudaia, L. Bousshine, H.F. Fihri, G. De Saxcé, Déformations élastoplastiques des métaux en présence du contact frottant : Applications à la mise en forme des structures en 3D, $5^{\mathrm{e}}$ RNJCP, Faculté Ben M'Sik, Casablanca, Maroc, 2006, pp. 63 
[17] E. Boudaia, L. Bousshine, H.F. Fihri, G. De Saxcé, Simulation numérique $3 \mathrm{D}$ du comportement élastoplastique des métaux en présence du contact avec frottement sec de Coulomb, $8^{\mathrm{e}}$ Congrès de mécanique, Faculté Chouaib Doukkali, El Jadida, Maroc, 2007, pp. $372-374$

[18] E. Boudaia, L. Bousshine, H.F. Fihri, G. De Saxcé, Elastoplastic deformations of metals in the presence of the unilateral contact with friction, Proceedings of International Symposium on Composites and Aircraft Materials, EST, Agadir, Morocco, May 22-24, 2007, pp. 6-69
[19] E. Boudaia, L. Bousshine, H. Fihri, G. De Saxcé, Numerical simulation of the elastoplastic behavior of materials in the presence of the unilateral contact with friction, Proceedings of Inter. Conf. on Smart materials and Adaptive Structures: Mathematical Modeling and Computation, Faculty of sciences and Technology, Tangier, Morocco, April 14-16, 2008, pp. 168-171

[20] E. Boudaia, L. Bousshine, G. De Saxcé, A. Chaaba, A meshless method analysis of elastoplastic contact problems with friction, Int. J. Appl. Mech., accepted

[21] J.S. Fleuret, Prise en main de CASTEM 2000 par l'exemple, CEA Saclay/DRN/DMT/SEMT/LAMS, 1996 